\title{
IGF1R signaling drives antiestrogen resistance through PAK2/PIX activation in luminal breast cancer
}

Article in Oncogene · January 2018

DOI: $10.1038 / 541388-017-0027-9$

CITATIONS

0

18 authors, including:

\section{Yinghui Zhang}

Leiden University

11 PUBLICATIONS 132 CITATIONS

SEE PROFILE

\section{Tamar Geiger}

Tel Aviv University

120 PUBLICATIONS 3,680 CITATIONS

SEE PROFILE
READS

57

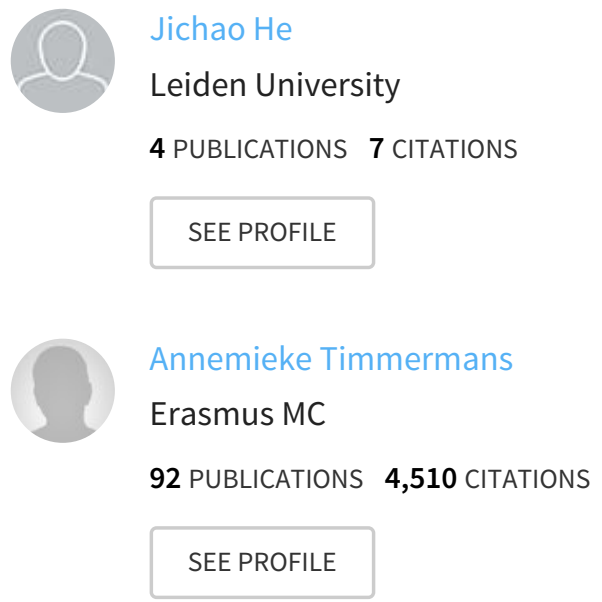

Some of the authors of this publication are also working on these related projects:

Project Recurrence of high grade serous ovarian cancer (HGSOC) View project

Project Carcinogenicity View project 


\title{
IGF1R signaling drives antiestrogen resistance through PAK2/PIX activation in luminal breast cancer
}

\author{
Yinghui Zhang ${ }^{1} \cdot$ Lynn Wester $^{1} \cdot$ Jichao He $^{1} \cdot$ Tamar Geiger $^{2} \cdot$ Marja Moerkens $^{1} \cdot$ Ram Siddappa $^{1} \cdot$ Jean A. Helmijr ${ }^{3}$. \\ Mieke M. Timmermans ${ }^{3}$ - Maxime P. Look ${ }^{3}$. Caroline H.M. van Deurzen ${ }^{3,4}$. John W.M. Martens $\mathbb{D}^{3}{ }^{3}$ Chantal Pont $^{1}$. \\ Marjo de Graauw ${ }^{1}$ - Erik H.J. Danen ${ }^{1}$ - Els M.J.J. Berns ${ }^{3}$ - John H.N. Meerman ${ }^{1}$ Maurice P.H.M. Jansen ${ }^{3}$. \\ Bob van de Water ${ }^{1}$
}

Received: 6 March 2017 / Revised: 31 August 2017 / Accepted: 24 September 2017

(c) Macmillan Publishers Limited, part of Springer Nature 2018

\begin{abstract}
Antiestrogen resistance in estrogen receptor positive $\left(\mathrm{ER}^{+}\right)$breast cancer is associated with increased expression and activity of insulin-like growth factor 1 receptor (IGF1R). Here, a kinome siRNA screen has identified 10 regulators of IGF1Rmediated antiestrogen with clinical significance. These include the tamoxifen resistance suppressors $B M P R 1 B, C D K 10$, $C D K 5, E I F 2 A K 1$, and MAP2K5, and the tamoxifen resistance inducers CHEK1, PAK2, RPS6KC1, TTK, and TXK. The p21activated kinase 2, $P A K 2$, is the strongest resistance inducer. Silencing of the tamoxifen resistance inducing genes, particularly PAK2, attenuates IGF1R-mediated resistance to tamoxifen and fulvestrant. High expression of PAK2 in ER ${ }^{+}$ metastatic breast cancer patients is correlated with unfavorable outcome after first-line tamoxifen monotherapy. Phosphoproteomics has defined PAK2 and the PAK-interacting exchange factors PIX $\alpha / \beta$ as downstream targets of IGF1R signaling, which are independent from PI3K/ATK and MAPK/ERK pathways. PAK2 and PIX $\alpha / \beta$ modulate IGF1R signaling-driven cell scattering. Targeting PIX $\alpha / \beta$ entirely mimics the effect of PAK2 silencing on antiestrogen re-sensitization. These data indicate PAK2/PIX as an effector pathway in IGF1R-mediated antiestrogen resistance.
\end{abstract}

Yinghui Zhang and Lynn Wester are contributed equally to this work

Electronic supplementary material The online version of this article (https://doi.org/10.1038/s41388-017-0027-9) contains supplementary material, which is available to authorized users.

Yinghui Zhang

y.zhang@lacdr.leidenuniv.nl

$\triangle$ Bob van de Water

b.water@lacdr.leidenuniv.nl

1 Division of Toxicology, Leiden Academic Center for Drug Research, Leiden University, Leiden, The Netherlands

2 Department of Human Molecular Genetics and Biochemistry, Sackler Faculty of Medicine, Tel Aviv University, Tel Aviv, Israel

3 Department of Medical Oncology and Cancer Genomic Netherlands, Rotterdam, The Netherlands

4 Department of Pathology, Erasmus MC Cancer Institute, Erasmus University Medical Center, Rotterdam, The Netherlands

\section{Introduction}

More than $70 \%$ of breast cancers are estrogen receptor positive $\left(\mathrm{ER}^{+}\right)$and dependent on estrogen for growth and progression [1]. Antiestrogen therapy by tamoxifen or fulvestrant has been the front-line treatment for $\mathrm{ER}^{+}$breast cancers $[2,3]$. Despite the effectiveness at first, around 50\% of the treated breast cancers will become antiestrogenresistant $[4,5]$. Antiestrogen resistance is attributed to loss of ER expression or function in breast cancers where ER does not actually play a central role [6, 7]. Additionally, resistant breast cancers often show upregulated signaling of receptor tyrosine kinases, including epidermal growth factor receptor [8, 9], HER2 [10, 11] and insulin-like growth factor 1 receptor (IGF1R) [12-14].

High IGF1R expression is associated with migration and invasion of breast cancer and metastasis-free survival of ER + breast cancer patients [15-19]. IGF1R activation by insulin-like growth factor 1 (IGF-1) promotes cell death resistance of $\mathrm{ER}^{+}$breast cancer cells [17]. During treatment of breast cancer, IGF1R expression is upregulated, resulting in resistance to conventional breast cancer therapies and 
poor outcome [20]. Several high-throughput screening studies have mapped IGF1R as a candidate target to resensitize tamoxifen resistant breast cancer cells [12, 21, 22]. We and others have demonstrated that IGF1R signaling can confer resistance to antiestrogen treatment regardless of ER activity $[12,14]$. IGF1R is coupled to multiple downstream pathways and, besides PI3K/AKT and MAPK/ERK signaling [14], it is unresolved which of these contribute to antiestrogen resistance.

Here we performed a small interfering RNA (siRNA) screen targeting all kinases and phosphatases to unravel signaling cascades underlying IGF1R-mediated antiestrogen resistance. For identified candidate targets to combat resistance, association was determined with clinical outcome of metastatic $\mathrm{ER}^{+}$breast cancers treated with first-line of tamoxifen monotherapy. Our results reveal PAK2, as well as the PAK-interacting exchange factors PIX $\alpha / \beta$, as a critical determinant in IGF1R-mediated antiestrogen resistance with significant clinicopathological correlation to tamoxifen resistance development.

\section{Results}

\section{Kinome siRNA screen of IGF1R-mediated tamoxifen resistance}

MCF7/IGF1R cells with ectopic IGF1R expression (Supplementary Fig. 1A) remain sensitive to the antagonistic effects of antiestrogens 4-hydroxytamoxifen (4OHT) and fulvestrant (FUL) against estrogen $17 \beta$-estradiol (E2), but are highly resistant when stimulated with IGF-1 (Supplementary Fig. 1B) [14]. Using this cell model, we performed a human kinase and phosphatase siRNA primary screen under the tamoxifen resistance condition, as schemed (Supplementary Fig. 2A). Replica screens displayed high reproducibility (Fig. 1a, b). Z-score ranking revealed 66 kinases and 8 phosphatases whose knockdown increased cell proliferation, and 79 kinases and 21 phosphatases whose targeting inhibited cell proliferation (Fig. 1a, b and Supplementary Table 1). We classified the former genes as tamoxifen resistance suppressors, meaning that their expression or signaling may suppress tamoxifen resistance, and the latter as tamoxifen resistance inducers, meaning that their expression or signaling induces tamoxifen resistance.

Next, we validated all tamoxifen resistance inducers and a few top ranked or clinically relevant tamoxifen resistance suppressors (as mentioned below), for their on-target silencing by SMARTpool siRNA and its 4 deconvoluted siRNAs and for their functional relevance to tamoxifen resistance status, compared to E2 and IGF-1 stimulation (Supplementary Fig. 2B). All validated 114 hits displayed positive effect under the resistance condition
(Supplementary Fig. 3A). The majority of hits were ontarget (Fig. 1c and Supplementary Fig. 4). Fifty-one hits were common to all conditions, while others were specific to one or two conditions (Fig. 1d and Supplementary Fig. 4). Particularly, several positive hits showed no effect or an opposite effect under IGF-1 or E2 condition, implying their distinct roles in IGF1R-mediated tamoxifen resistance (Supplementary Fig. 3B and C). These included Abll, CKM, MAP4K3, NTRK2, PAK2, RPS6KC1, SAST, SSTK, $T A F 1, P P P 2 R 5 D$, and TPTE2.

\section{Clinical relevance of IGF1R-mediated tamoxifen resistance hits}

To evaluate the clinical relevance of the screened hits (Supplementary Table 1), we analyzed them as continuous variable in our established Agilent 44k oligo-array dataset of $101 \mathrm{ER}^{+}$metastatic breast cancer (MBC) patients treated with first-line tamoxifen monotherapy [23, 24]. All candidate genes in this dataset could be analyzed for an association with treatment outcome. The results revealed 10 hits with high relevance to poor treatment outcome and progression free survival (PFS) (Fig. 2a). Significantly, the predicted hazard ratio (HR) is low in patients expressing tamoxifen resistance suppressors $B M P R 1 B, C D K 10, C D K 5$, $E I F 2 A K 1$, and $M A P 2 K 5$, and high in those expressing tamoxifen resistance inducers CHEK1, PAK2, RPS6KC1, $T T K$, and $T X K$. Furthermore, we evaluated these 10 hits for their prognostic values with the natural course of disease in $221 \mathrm{ER}^{+}$lymph node negative (LNN) breast cancer patients who did not receive any adjuvant systemic treatment [25]. The results demonstrated that expression levels of BMPR1B, CDK10, EIF2AK1, MAP2K5, CHEK1, and $P A K 2$, were not prognostic in this subset (Fig. 2b), showing a relationship only with tamoxifen therapy response.

\section{Targeting of tamoxifen resistance inducing hits with clinical relevance restores antiestrogen sensitivity}

These 10 hits were on-target under tamoxifen resistance condition, displaying differential effects under IGF-1 or E2 stimulative condition (Fig. 1e). Next, we established their dose-response relationships to 4OHT (Fig. 3a) and FUL (Fig. 3b) in combination with E2 and IGF-1. Compared to siRNA control (siCtrl), silencing of each tamoxifen resistance suppressor enhanced proliferation by IGF-1 and $\mathrm{E} 2$, above the siCtrl levels where the resistance remains. In contrast, silencing of the tamoxifen resistance inducer CHEK1, TTK, or TXK significantly reduced IGF-1 and E2 induced proliferation, largely below the siCtrl levels. Of interest, targeting the inducer $P A K 2$ or $R P S 6 K C 1$ restored the dose-dependent response to 4OHT and FUL, in the presence of IGF-1. 
a

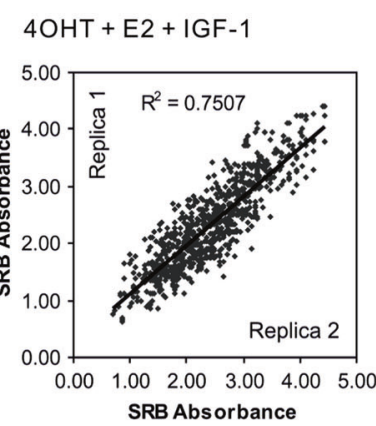

b

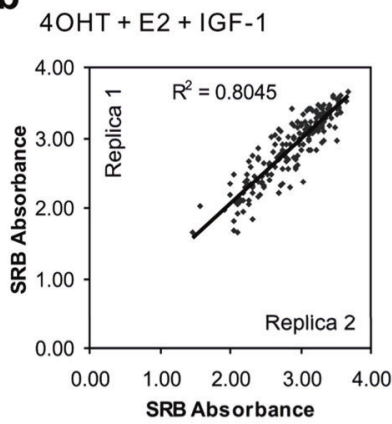

d

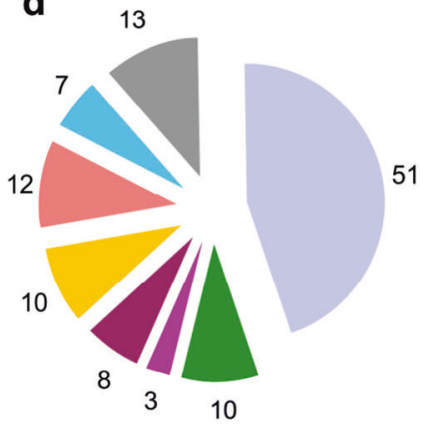

- on-target under condition I, II, III non-target under condition I only चon-target under condition II only an-target under condition III only an-target under condition I, II - on-target under condition I, III " on-target under condition II, III च off-target under condition I, II, III
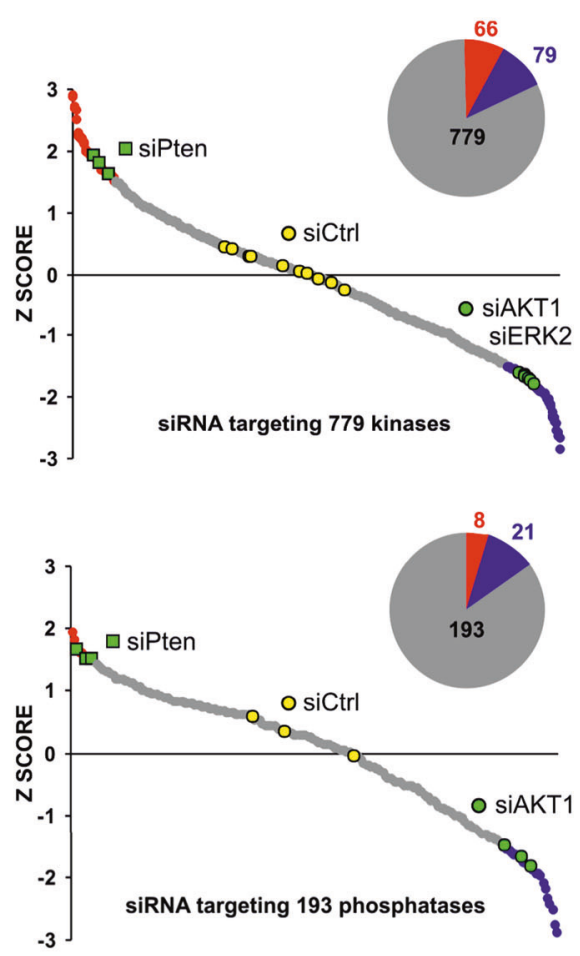

siRNA_1, 22,_3,_4 individuals

114 (91 kinases +23 phosphatases)

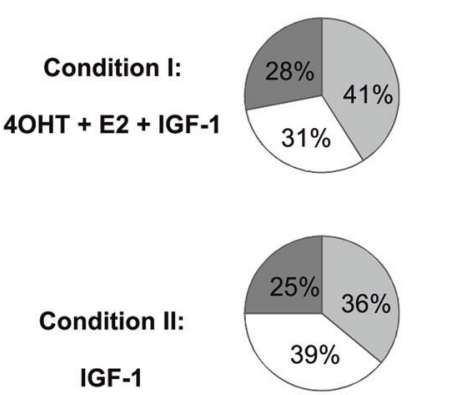

Condition III:

E2

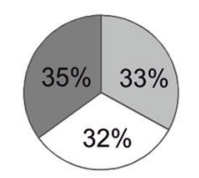

$4 / 4$, on-target hits

$3 / 4$, on-target hits

$<=2 / 4$, off-target

e

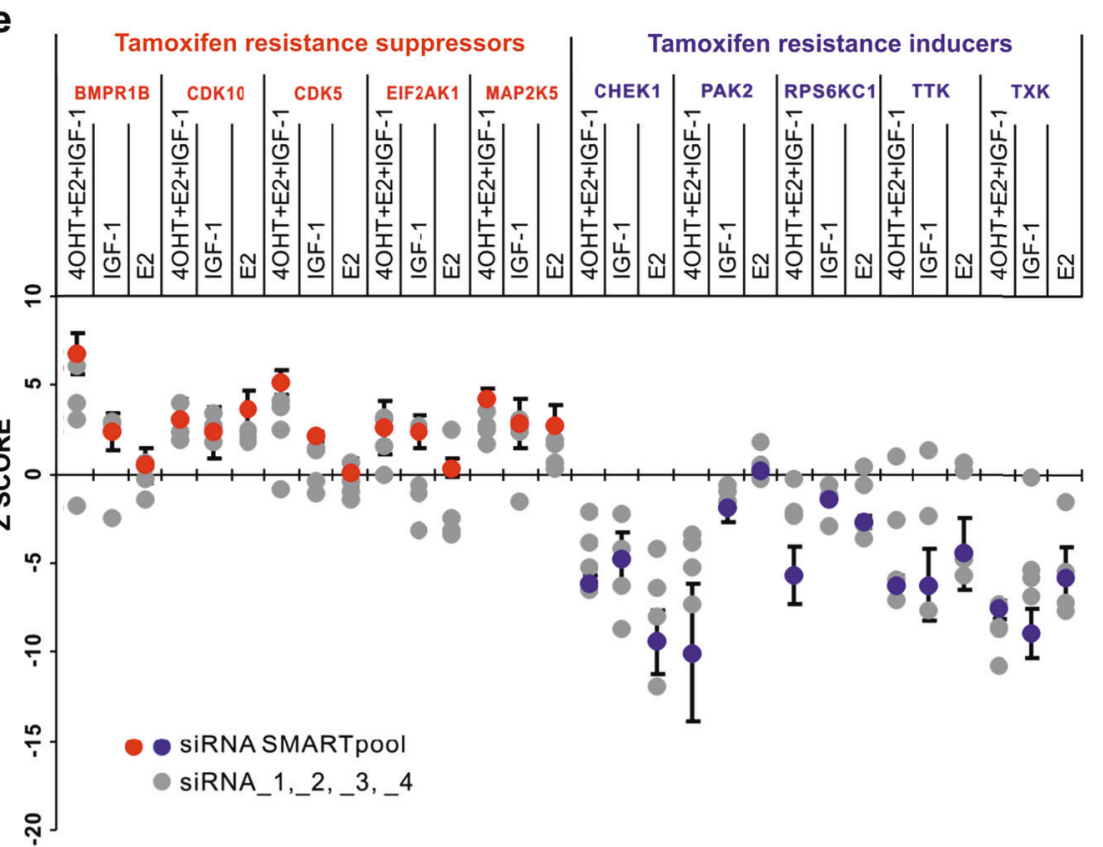

Fig. 1 Identification of determinants in IGF1R-mediated tamoxifen resistance by kinome siRNA Screen. a Primary kinase and b phosphatase siRNA screen of MCF7/IGF1R cells under tamoxifen resistance condition where 4OHT (4-hydroxytamoxifen, $1 \mu \mathrm{M})$, E2 (17 $\beta$ estradiol, $1 \mathrm{nM}$ ) and IGF-1 (insulin-like growth factor 1, $100 \mathrm{ng} / \mathrm{ml}$ ) were given. Spearman correlation coefficient $\mathrm{R}^{2}$ values showed reproducibility of replica screens. The hits for tamoxifen resistance suppressors $(Z>$ mean $+1.5 \times \mathrm{SD}, P<0.05$; in red) and inducers $(Z$ $<$ mean $-1.5 \times \mathrm{SD}, P<0.05$; in blue) were ranked by $Z$ score plot and numbered in pie charts. siRNAs targeting PTEN, AKT1, and MAPK1, the known regulators in IGF1R-mediuated antiestrogen resistance [14], were used as functional positive controls (Supplementary Figs. 1C and
D). The targeting effects of siPten (increasing proliferation), siAKT1 and siERK2 (decreasing proliferation), and the non-targeting effect of negative control siCtrl were positioned in the $Z$ score plots. c Validation screen of IGF1R-mediated tamoxifen resistance hits. Effects of single siRNAs (siRNA_1, _2,_3 and _4) targeting 114 hits were validated under tamoxifen resistance condition (I) 4OHT + E2 + IGF1, compared to IGF-1 (II) and E2 (III) stimulation conditions. d The number of hit siRNAs showing on-target effects under condition I, II and III. e Comparison of SMARTpool siRNA and single siRNA_1, _2, 3 and $\_4$ targeting effects on 10 hits, which have clinical relevance (Fig. 2 a), under condition I, II and III in repeated experiment. Values were derived from triplicate samples. Data were expressed as mean $\pm \mathrm{SD}$ 
a mRNA levels related to tamoxifen treatment outcome in 101 metastatic breast cancer patients

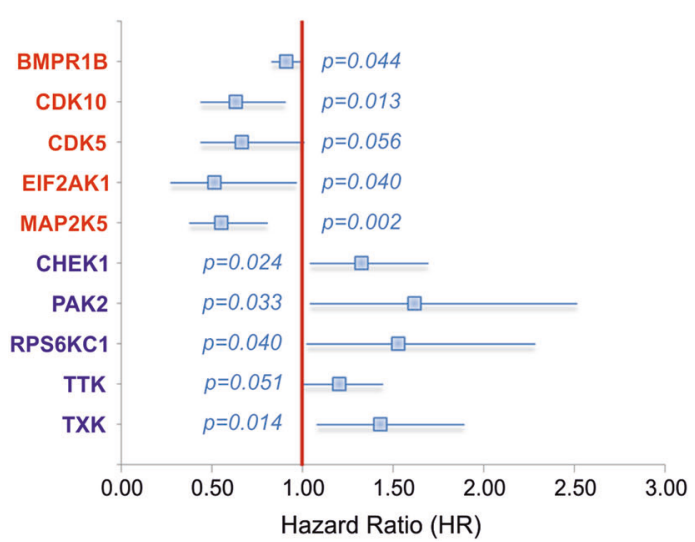

Fig. 2 Clinical relevance of 10 IGF1R-mediated tamoxifen resistance hits The hazard ratios of the 10 hits are indicated by blue boxes and their $95 \%$ confidence intervals $(95 \% \mathrm{CI})$ are represented by blue horizontal lines. The $P$-value is shown for those significant. a The 10 hits and the relationship with PFS in 101 breast cancer patients with metastatic disease treated with first-time tamoxifen monotherapy,

\section{PAK2 is a target in IGF1R-mediated antiestrogen resistance}

Our transcriptomic data revealed the high expression of PAK2 in both parental MCF7 and the established MCF7/ IGF1R cells, compared to any other PAK family members, suggesting a critical role for PAK2 in these cells (Supplementary Fig. 5A-B). Silencing of PAK2 (siPAK2), but none of the other PAK members that are expressed in the MCF7/ IGF1R cells, PAK1, PAK4 and PAK6 (Supplementary Fig. 5C-D), inhibited cell proliferation significantly under tamoxifen resistance condition, less so in IGF-1 stimulation or not at all under E2 condition (Fig. 1e), and restored antiestrogen dose-responses (Fig. 3a, b). PAK2 predicated the highest HR in tamoxifen-treated patients (Fig. 2a), leading to poor survival (Fig. 4a). These results let us first focus on the potential role of $P A K 2$, p21 protein (Cdc42/ Rac)-activated kinase 2, in IGF1R-mediated antiestrogen resistance.

PAK2 on-target silencing re-sensitized MCF7/IGF1R cells (Fig. 4b). Similar to siCtrl, siPAK2 allowed 4OHT and FUL to antagonize E2 through a dose range, and did not significantly influence IGF-1-stimulated proliferation. Yet, siPAK2 declined the proliferation of the resistant cells under 4OHT or FUL dose-range with E2 plus IGF-1, reaching similar levels under 4OHT/E2 or FUL/E2 antagonizing condition (Fig. 4c).

Lentiviral shRNA-mediated PAK2 stable knockdown in MCF7/IGF1R cells, shPAK2_1 and shPAK2_2, did not interfere with ER expression (Fig. 4d), E2 and IGF-1 stimulated proliferation, or the antagonistic effects of $4 \mathrm{OHT}$ and FUL on E2 (Supplementary Fig. 6). Nonetheless, while

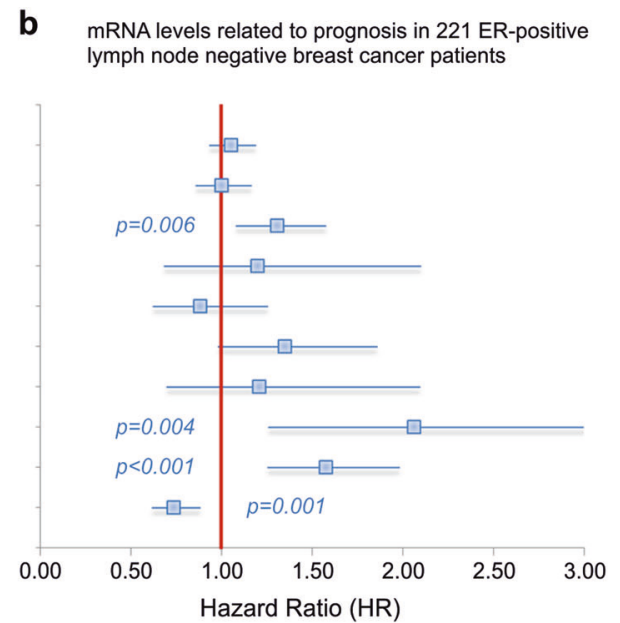

when analyzed as continuous variable. Hits in red, tamoxifen resistance suppressors, with low Hazard Ratio $(\mathrm{HR}<1)$. Hits in blue, tamoxifen resistance inducers, with high HR $(>1)$. b The 10 hits and the relation with metastasis free survival in $221 \mathrm{ER}^{+}$breast cancer patients who did not receive any adjuvant systemic therapy. This patient cohort has been described by us previously [25]

IGF-1 provoked resistance in shRNA control (shCtrl) cells, the resistant phenotype was drastically abolished in shPAK2 cells (Supplementary Fig. 6) in 4OHT and FUL concentration-dependent manners (Fig. 4e). As expected, rescue of PAK2 expression in shPAK2 cells (Fig. 4f) rendered the cells resistant to 4OHT and FUL (Fig. 4g). These results indicated a pivotal role of PAK2 in controlling IGF1R-mediated antiestrogen resistance.

\section{PAK2 acts downstream of IGF1R signaling independently from PI3K/AKT and MAPK/ERK pathways}

Relatively little is reported about activation of PAK signaling in response to IGF-1 stimulation. To decipher IGF-1/ IGF1R assembled signaling networks, we performed a Stable-Isotope Labeling by Amino Acids in Cell Culture (SILAC)-based quantitative phospho-proteomics, as schemed (Fig. 5a). Quantitative SILAC-based analysis revealed that a number of components in IGF-1 and PAK signaling were highly phosphorylated upon IGF-1 stimulation (Fig. 5b). Ingenuity Pathway Analysis (IPA) plotted out the crosstalk between the IGF-1 and PAK signaling networks, where the signaling components $A T M, M A P 2 K 1$, MAP2K2, MAPK1, MAPK3, PIK3C2A, PXN, RAF1, SHC1, and $S O S 1$ were activated (Fig. $5 \mathrm{c}$ ).

IGF-1 stimulation induced the phosphorylation of numerous proteins/kinases that regulate cell proliferation, growth and survival programs downstream of IGF1R signaling pathway as mapped by IPA (Fig. 6a.). These kinases mostly contributed as inducers to IGF1R-mediated tamoxifen resistance, including IGFIR, PI3K catalytic subunits 
a $\quad 4 \mathrm{OHT}+\mathrm{E} 2+\mathrm{IGF}-1$

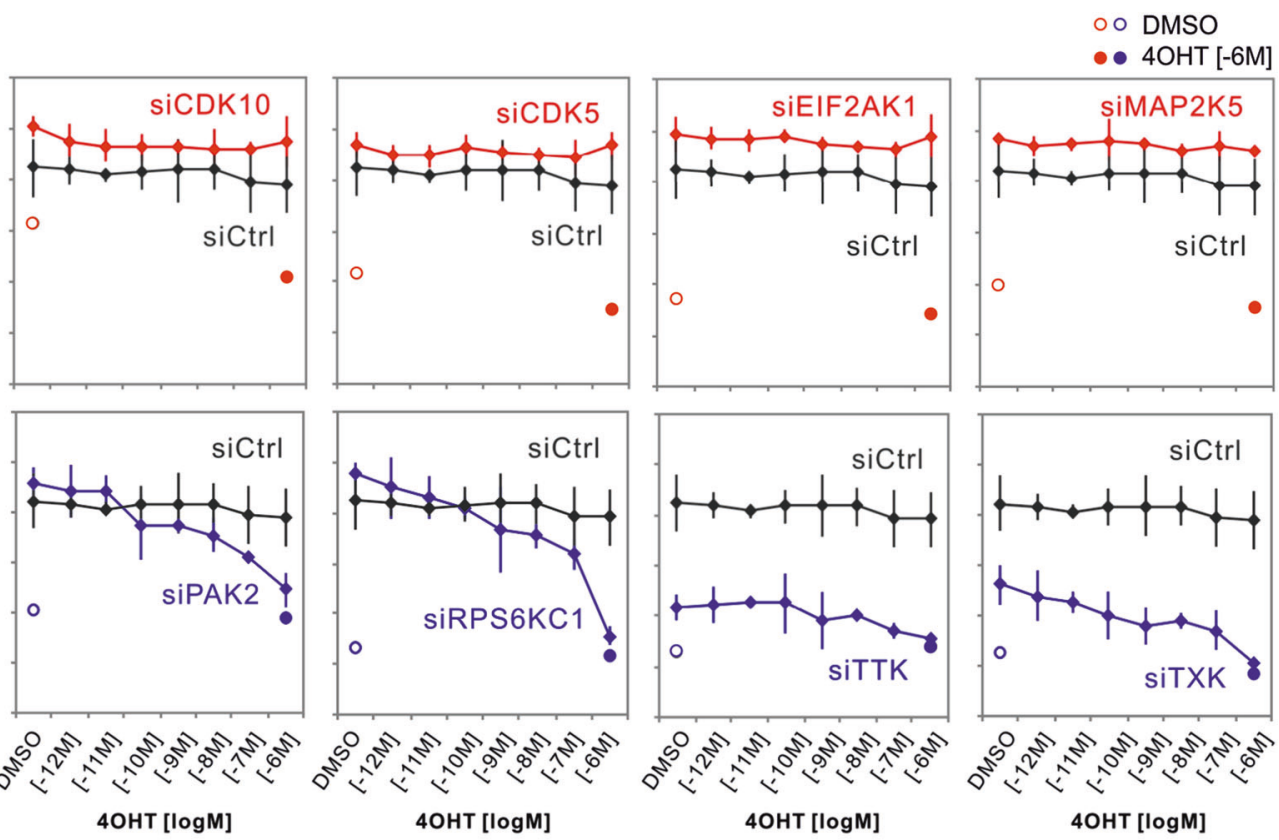

b $\mathrm{FUL}+\mathrm{E} 2+\mathrm{IGF}-1$

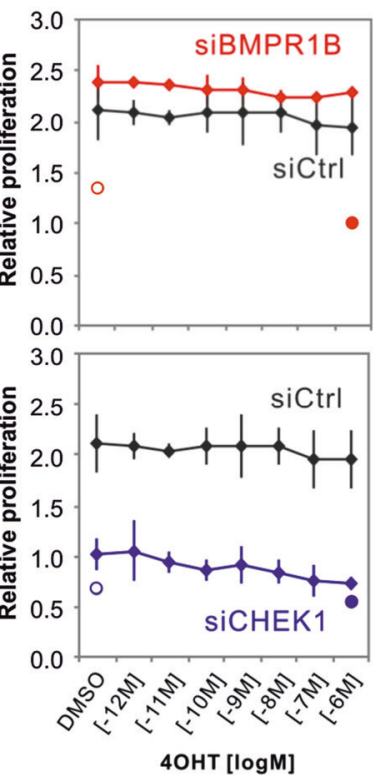

4OHT [ $\log M]$

4OHT [ $[\log M]$

4OHT [ $\log M]$
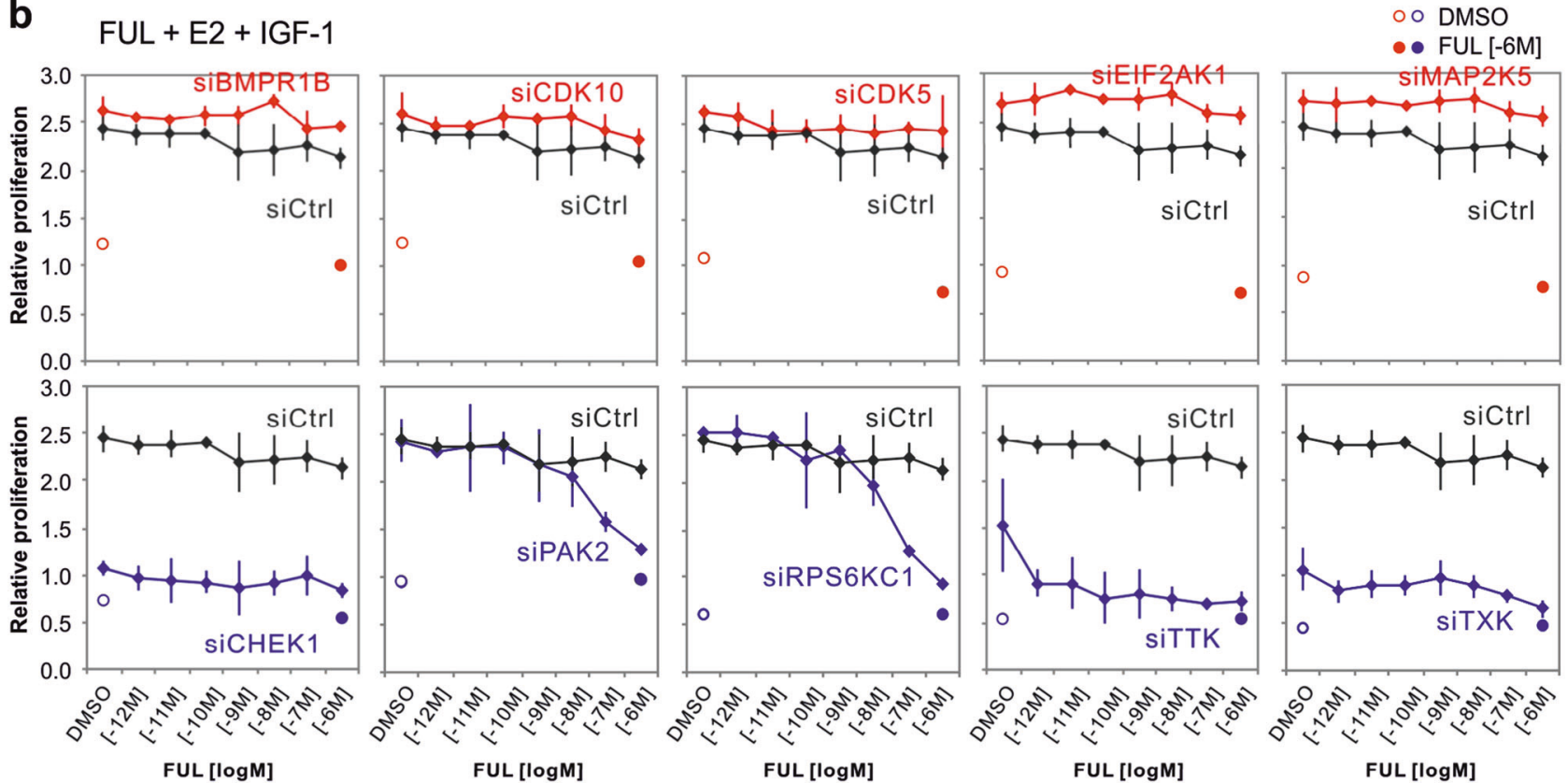

Fig. 3 Targeting effects on antiestrogen dose-responses of the IGF1Rmediated tamoxifen resistance hits with clinical relevance. a 4OHT and b FUL dose-response curves of siRNA silenced hits. FUL,

$(P I K 3 C A$ and $P I K 3 C 2 G), P D K 1, A K T 1, \quad$ p70S6Ks (RPS6KA2, RPS6KB1 and RPS6KC1), Rafl and MAPK1 (Supplementary Fig. 5E). Importantly, SILAC spectra revealed PAK2 phosphorylation at Ser141 (Fig. 6b). This IGF-1-stimulated PAK2 phosphorylation was rapid and constant (Fig. 6c and Supplementary Fig. 7A). IGF1R kinase inhibitor BMS-538924 blocked PAK2 fulvestrant. DMSO, vehicle control. E2, $1 \mathrm{nM}$; IGF-1, $100 \mathrm{ng} / \mathrm{ml}$. Proliferation values were relative to siCtrl under DMSO control. Values were derived from triplicate samples. Data were expressed as mean $\pm \mathrm{SD}$

phosphorylation (Fig. 6d, left panels), confirming PAK2 signaling in IGF-1/IGF1R axis.

PAK signaling is linked to Grb2-Raf-ERK and PI3K/ AKT (Fig. 6a) [26-30], the two canonical routes downstream of receptor tyrosine kinase signaling transduction, which are interrelated with IGF-1 and PAK signaling networks (Fig. 5c). We showed here that PAK2, 
a

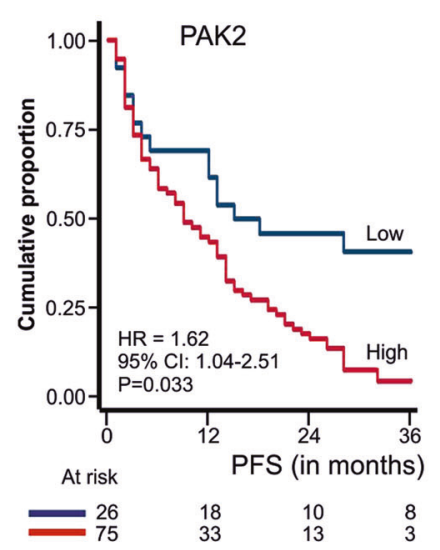

b

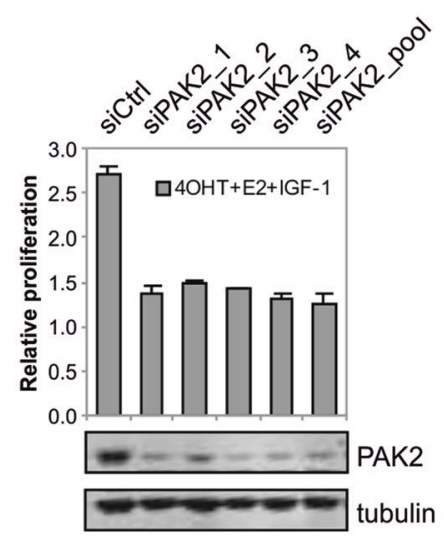

e

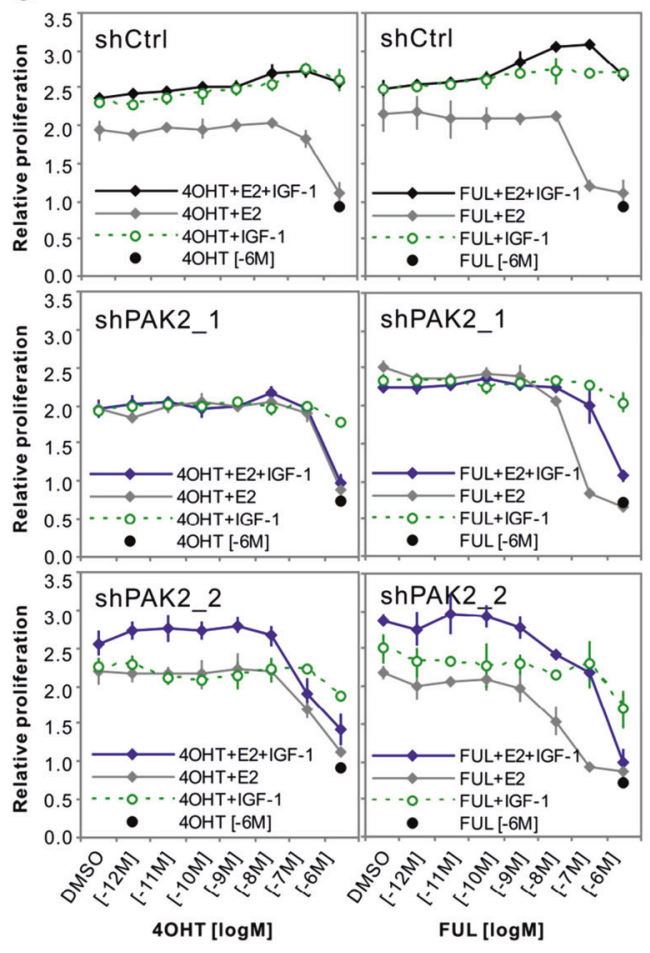

d
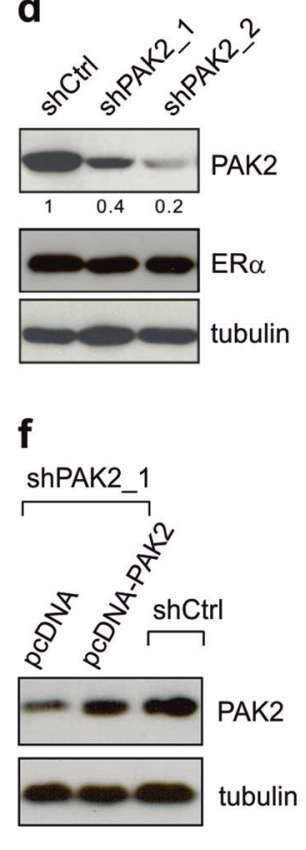

C
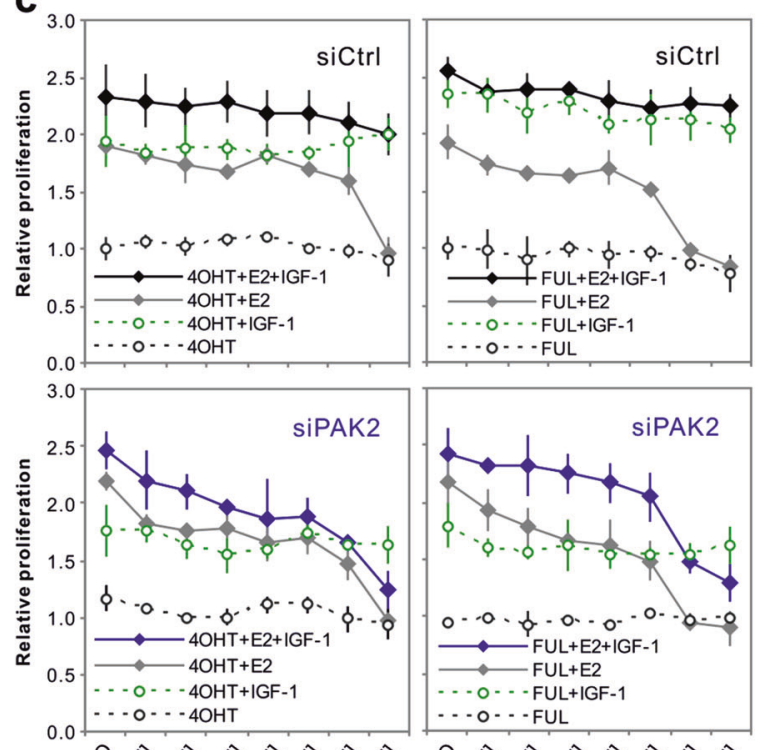

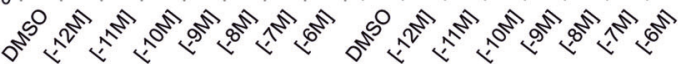
$4 \mathrm{OHT}[\log \mathrm{M}]$

FUL $[\log M]$

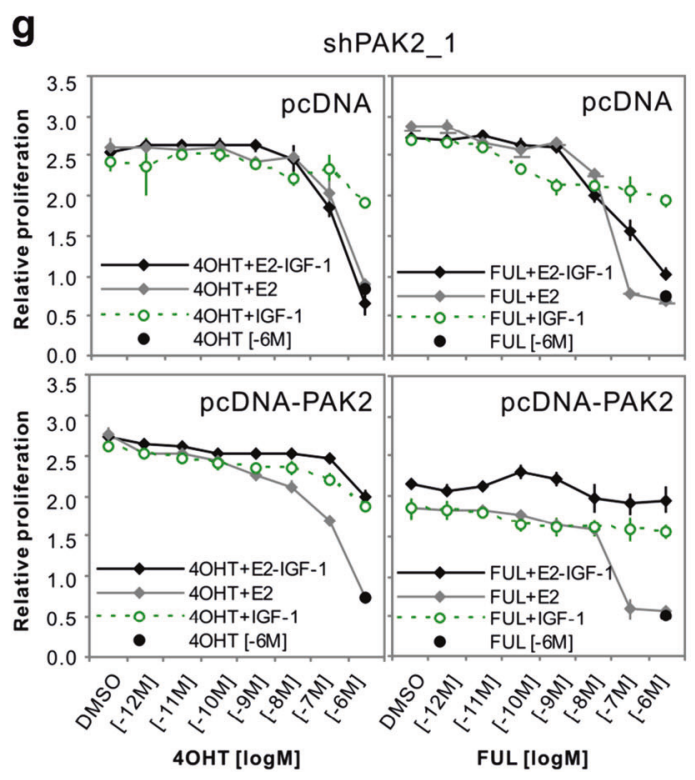

Fig. 4 Targeting of tamoxifen resistance inducer PAK2 abrogates IGF1R-mediated antiestrogen resistance. a Kaplan-Meier survival curve for PFS in relation to PAK2 RNA expression level in metastatic breast cancers treated with first-line tamoxifen monotherapy, showing the poor survival in 75 patients with high level of PAK2. b Inhibitory effects of PAK2 SMARTpool and single siRNAs on PAK2 expression and proliferation under tamoxifen resistance condition 4OHT $(1 \mu \mathrm{M})$ + E2 $(1 \mathrm{nM})+$ IGF-1 $(100 \mathrm{ng} / \mathrm{ml})$. c Response curves of siPAK2, compared to siCtrl, to dose-ranged 4OHT or FUL with E2 $(1 \mathrm{nM})$ and/ or IGF-1 $(100 \mathrm{ng} / \mathrm{ml})$, as indicated. Proliferation values were relative to siCtrl under DMSO control. d Lentivirus-mediated small hairpin RNA

simultaneously with AKT and ERK, was activated by IGF1 stimulation (Supplementary Fig. 7A). While siPAK2 or shPAK2 depletion did not eliminate ERK and AKT phosphorylation (Fig. 6e), MEK inhibitors (U0126, PD-184352
(shRNA) stable knockdown of PAK2 in MCF7/IGF1R shPAK2_1 and shPAK2_2 cells, compared to endogenous PAK2 in shRNA control (shCtrl) cells. e Dose-dependent responsiveness of shPAK2_1 and shPAK2_2 cells to 4OHT and FUL under indicated conditions, compared to shCtrl control cells. Proliferation values were relative to DMSO control. f PAK2 cDNA expression in shPAK2_1 cells. g Recurrent resistance of shPAK2 cells with rescued PAK2 cDNA expression to 4OHT and FUL under indicated conditions, compared to pcDNA vector transfected shPAK2 cells. Proliferation values were relative to DMSO control. Values were derived from triplicate samples. Data were expressed as mean $\pm \mathrm{SD}$

and AZD6244) and PI3K/AKT inhibitors (BEZ235 and PI103) had no effect on PAK2 activation (Fig. 6d, middle and right panels). Similarly, AKT silencing did not inhibit IGF-1-stimulated PAK2 phosphorylation (Supplementary 
Fig. 5 PAK signaling in response to IGF-1 stimulation and interaction with IGF-1 signaling network. a Quantitative SILACbased phospho-proteomics scheme of MCF7/IGF1R cells under IGF-1 stimulation. Parental MCF7 cell line was taken as control. IGF-1, $100 \mathrm{ng} / \mathrm{ml}, 30$ min. b IPA reported phosphorylated components in IGF-1 and PAK signaling pathways. Phosphorylation level was given as ratio of IGF-1 stimulated ("Medium" labeling) to nonstimulated ("Heavy" labeling) MCF7/IGF1R cells. c IPA mapped interaction of phosphorylated components in IGF-1 and PAK signaling networks. Components in red, cross-talking in IGF-1 and PAK signaling networks a
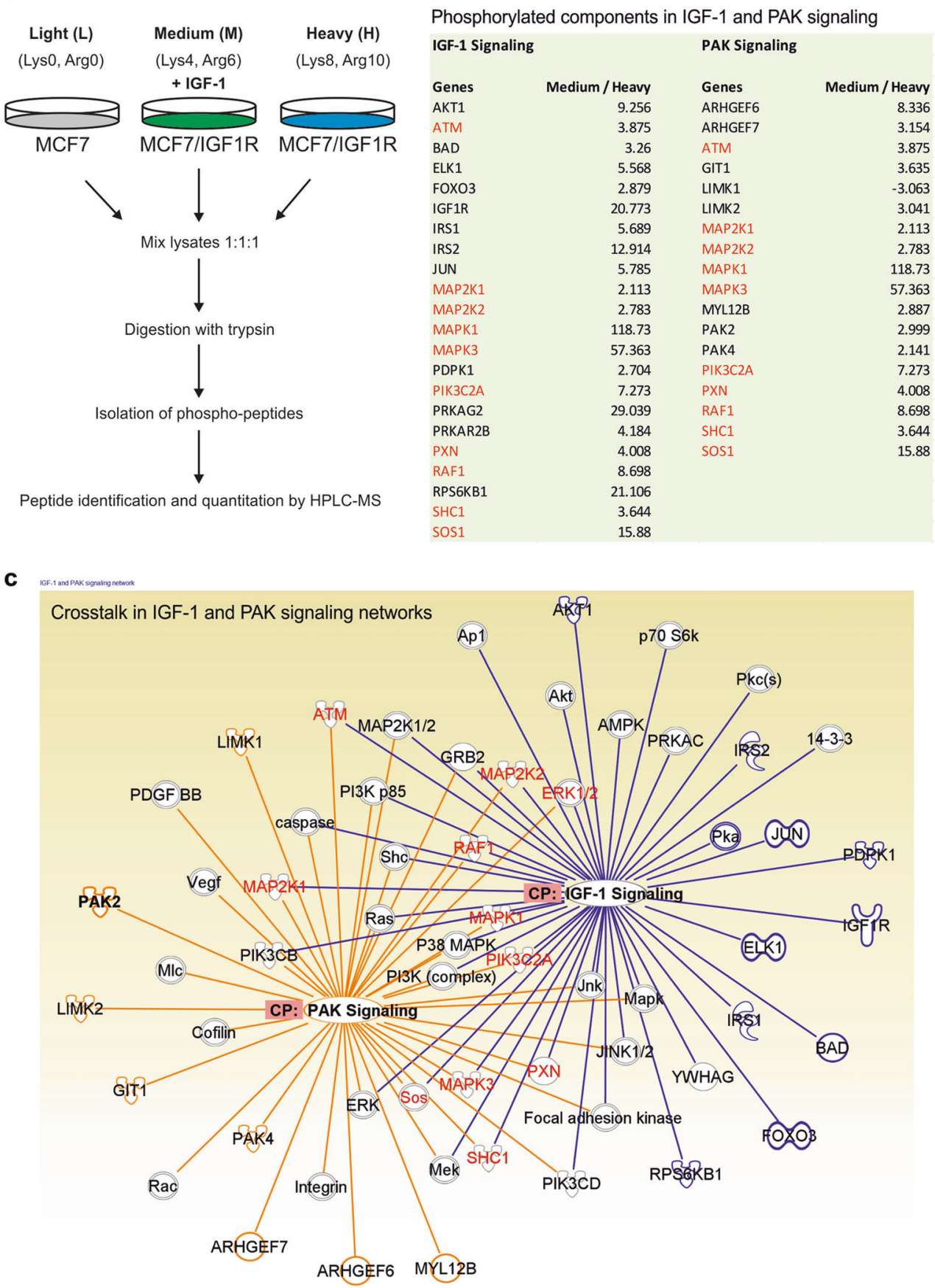

Fig. 7B). These results delineated that PAK2 signaling deviates from PI3K/AKT and MAPK/ERK pathways in IGF-1/IGF1R axis.

\section{PAK2 suppresses the onset of apoptosis in IGF1R- mediated antiestrogen resistance}

PAKs play essential roles in cell-cycle progression and apoptosis prevention [26, 28, 31]. Next, we examined whether PAK2 modulates the cell cycle progression as well as the suppression of apoptosis in response to IGF-1 stimulation. IGF-1 promoted cell-cycle progression (G2/M and $\mathrm{S}$ phase) under 4OHT/E2 antagonizing condition, which was suppressed by shPAK2 (Fig. 6f and Supplementary Fig. 8A). This suppression coincided with an onset of apoptosis evidently at late time point, which also occurred under 4OHT/E2 (Fig. 6g and Supplementary Fig. 8B). We further evaluated this antiapoptotic effect of PAK2 
a

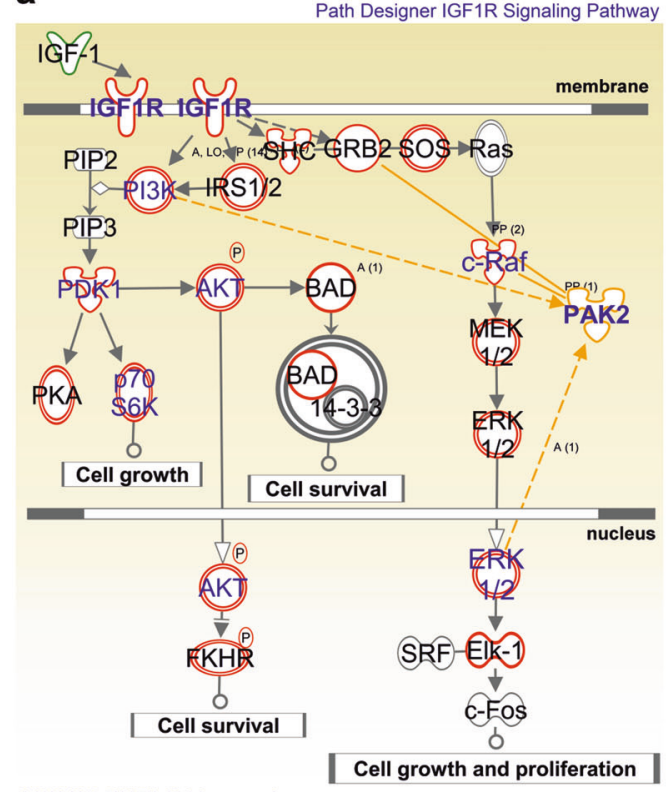

๑2000-2015 QIAGEN. All rights reserved b

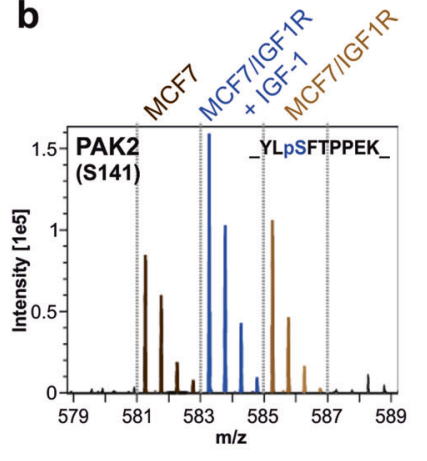

C

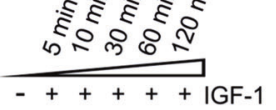

p-IGF1R (Y1131)

- =-2-IGF1R (Y1135/36)

IGF1Rß (total)

- - - D-PAK2 (S141)

PAK2 (total)

$\longrightarrow$ tubulin

e

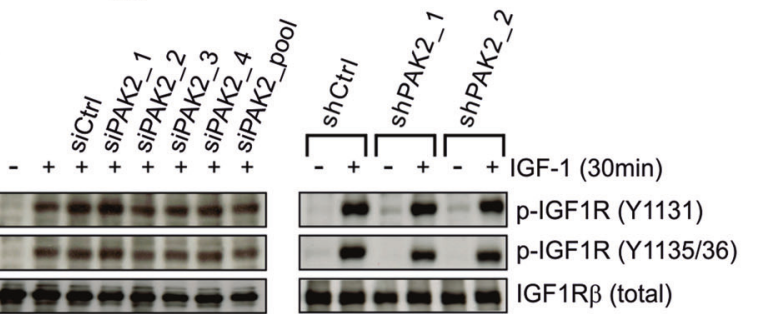

$--1+12$

$-\infty-\ldots$

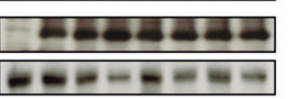

D-PAK2 (S141)

PAK2 (total)

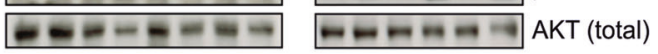

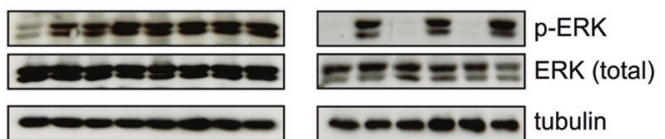

0.

pre-treat $2 h$ IGF-1 (30 min)

- - D-O-IGF1R (Y1131)

p-IGF1R (Y1135/36)

o-

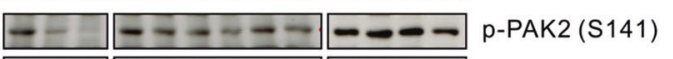

PAK2 (total)

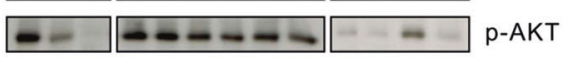

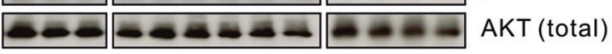

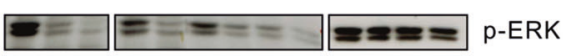

ERK (total)

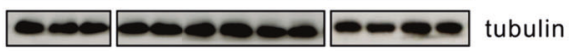
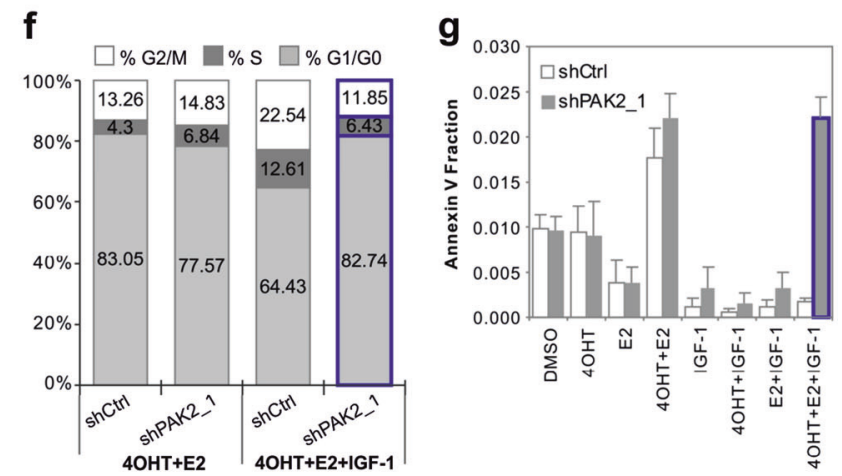

h

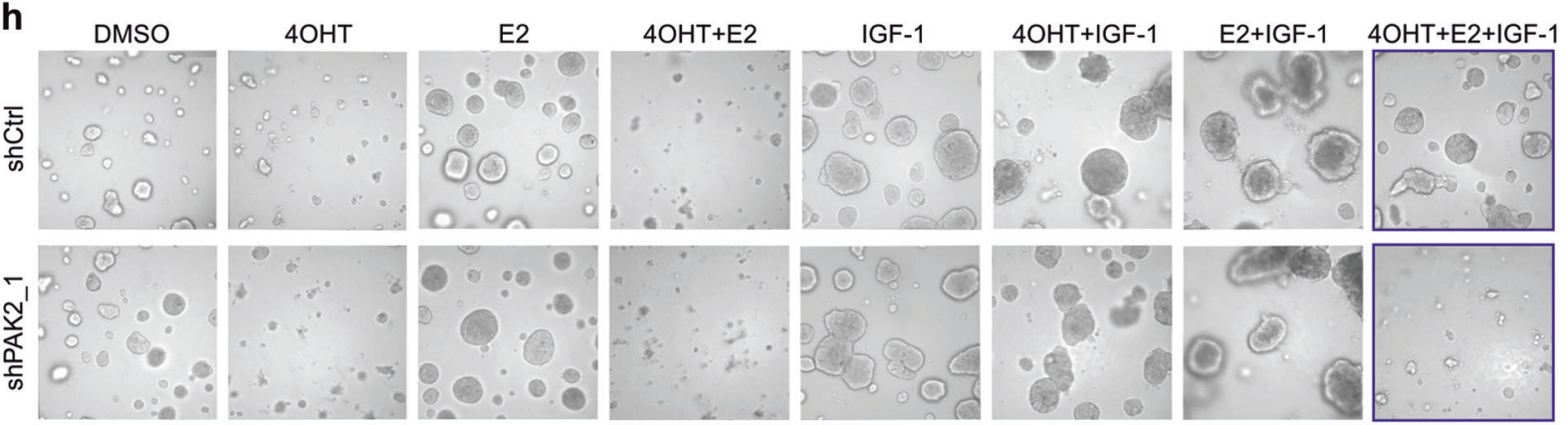


Fig. 6 PAK2 is activated apart from PI3K/AKT and MAPK/ERK pathways and promotes cell-cycle progression and anti-apoptosis in IGF1R-mediated antiestrogen resistance. a Ingenuity pathway analysis of SILAC phospho-proteomic data diagramed phosphorylated proteins/kinases (in red circles) in IGF-1/IGF1R signaling pathway. Tamoxifen resistance inducers were indicated (blue). The known relations of PAK2 to PI3K-AKT and GRB2-Raf-ERK downstream of IGF1R pathway were connected (yellow lines). b SILAC phosphoproteomics identified a high phosphorylation of PAK2 at Ser141 in IGF-1 stimulated MCF7/IGF1R cells. Peaks colored in gray, blue and brown represent the phosphopeptides from light-, medium- and heavyisotope labeled cells, respectively. c PAK2 phosphorylation in MCF7/ IGF1R cells in time course exposure to IGF-1 (100 ng/ml). d PAK2 phosphorylation status under inhibitory condition of IGF1R kinase inhibitor BMS-536924, MEK inhibitors U0126, PD-184352 and AZD6244, or PI3K inhibitors BEZ235 and PI-103, in IGF-1 stimulated MCF7/IGF1R cells. e IGF-1 induced phosphorylation of AKT and ERK in siPAK2 and shPAK2 depletion. f Flow cytometry sorting of cell proportions in cell-cycle G1/G0, G2/M and S phases in shCtrl and shPAK2 cells under 4OHT $+\mathrm{E} 2$ antagonizing versus $4 \mathrm{OHT}+\mathrm{E} 2$ + IGF-1 resistant condition. g Annexin V apoptotic fractions in shCtrl and shPAK2 cells under the 4-day treatments as indicated. Values were derived from triplicate samples. Data were expressed as mean \pm SD. h 3D culture showing acini formation of shCtrl and shPAK2 cells in 14 days responding to indicated treatments. Image $\times 20$

on IGF1R-mediuated resistance to $4 \mathrm{OHT}$ in a $3 \mathrm{D}$ culture system. While shCtrl cells formed acini under resistance condition, shPAK2 sufficiently abolished the resistant acinar outgrowth (Fig. 6h and Supplementary Fig. 8C).

\section{IGF1R-mediated antiestrogen resistance involves PAK2/PIX survival components}

PAK2 drives PAK-interacting exchange factor (PIX) activation in modulating cell migration and protrusion, promoting cell growth and survival, and preventing apoptosis [28, 32-34]. As a Rac effector, PAK2 is also essential for the activation of $\beta$-catenin [35], and the cell-cell adhesion protein $\beta$-catenin has been shown to mediate IGF-1/IGF1R action in cell proliferation [36]. We found that overexpression of IGF1R in MCF7 cells led to reduced cell-cell junctions (Supplementary Fig. 9A) and conferred a scattering phenotype in IGF-1 stimulation, which was diminished by shPAK2 (Fig. 7a). SILAC spectra revealed increased phosphorylation levels of PIX $\alpha / \beta$ (also named Rac/Cdc42 guanine nucleotide exchange factor 6/7, ARHGEF6/7) (Fig. 5b and Fig. 7b) as well as $\beta$-catenin (Supplementary Fig. 9E) after IGF-1 stimulation. Membranous staining of the cell-cell adhesion marker E-cadherin showed that silencing of PAK $2, \mathrm{PIX} \alpha / \beta$ or $\beta$-catenin inhibited the IGF1-mediated cell scattering phenotype (Fig. 7c and Supplementary Fig. 9B) and blocked IGF-1-modulated cell protrusion significantly (Fig. 7d). Furthermore, targeting PIX $\alpha$, PIX $\beta$ or $\beta$-catenin (Fig. 7e) recovered the sensitivity of MCF7/IGF1R cells to antiestrogens 4OHT and FUL (Fig. 7f), as did siPAK2, in dose-dependent manners
(Supplementary Fig. 9C and D). The effect of PAK2 was not limited to MCF7/IGF1R cells. Parental MCF7 cells express low level of endogenous IGF1R (Supplementary Fig. 1A) and demonstrate a limited IGF-1-mediated antiestrogen resistance (Supplementary Fig. 1B). Yet, importantly, also silencing of PAK2, PIX $\alpha, \operatorname{PIX} \beta$ or $\beta$-catenin in parental MCF7 decreased the proliferation under 4OHT or FUL, E2, and IGF-1 resistance conditions (Supplementary Fig. 9F). The involvement of PAK2 (but not other PAK family members), PIX $\alpha / \beta$ and $\beta$-catenin in IGF1R-mediated antiestrogen resistance was also confirmed in T47D/IGF1R, another established $\mathrm{ER}^{+}$breast cancer cell line with ectopic IGF1R overexpression (Supplementary Fig. 10A-E). Similar to $\operatorname{siPAK} 2, \operatorname{siPIX} \alpha, \operatorname{siPIX} \beta$ or $\beta$-catenin did not restrict IGF-1-stimulated AKT and ERK phosphorylation in both MCF7/IGF1R (Fig. 7g) and T47D/IGF1R cell lines (Supplementary Fig. 10F). While PAK1 has been shown to stabilize $\beta$-catenin [37], depletion of PAK2 did not affect $\beta$ catenin expression levels in neither MCF7/IGF1R (Fig. 7h) nor T47D/IGF1R (Supplementary Fig. 10G). Of relevance, IGF-1-induced PIX $\beta$ phosphorylation was downstream of IGF1R/PAK2 signaling, as siPAK2 inhibited the phosphorylation of PIX $\beta$ in IGF-1 stimulation in both resistant cell lines (Fig. 7h and Supplementary Fig. 10G). These data indicate PAK2/PIX signaling as a distinct signaling branch downstream of IGF1R activation. Importantly, the PAK2/ PIX-mediated antiestrogen resistance after IGF1R signaling seems to be a general phenomenon.

\section{PAK2 expression is associated with clinicopathological characteristics and progression free survival}

Our clinical dataset of $101 \mathrm{MBC}$ patients treated with firstline tamoxifen revealed that PAK2 RNA expression level predicated high HR in disease progression (Fig. 2a) and poor outcome as verified in Kaplan-Meier survival curve (Fig. 4a). To further assess the clinical relevance of PAK2, we next performed a PAK2 tissue microarray (TMA) staining in 291 patients with primary operable $\mathrm{ER}^{+}$breast cancer who subsequently developed MBC after first-line tamoxifen and for which detailed clinical follow-up was available [38]. TMA results showed that PAK2 protein staining was detected primarily in cytoplasm in 242 of the $291 \mathrm{ER}^{+}$tumors $(83 \%)$. The quantity of positive cells was indistinguishable, since almost all tumor cells within the evaluated biopsy cores were PAK2 positive. Staining intensity separated the specimens into 114 with weak staining (47\%), 92 with moderate staining (38\%), and 36 with strong staining (15\%) tumors (Fig. 8a, b). No significant associations were observed between PAK2 intensity and traditional predictive factors, including age and menopausal status at start of therapy, lymph nodes involved, 


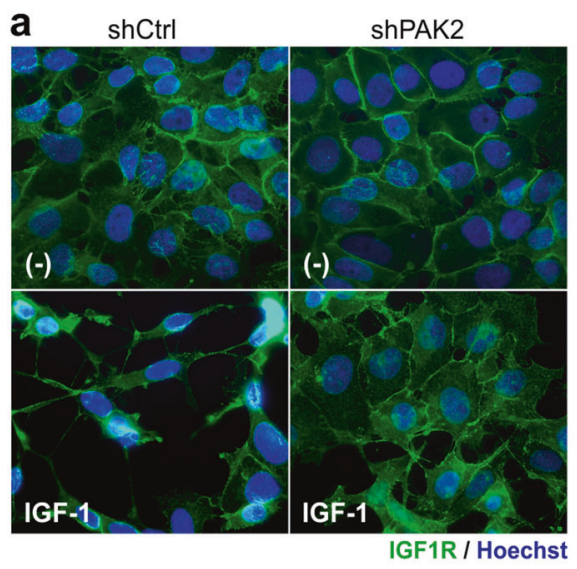

e

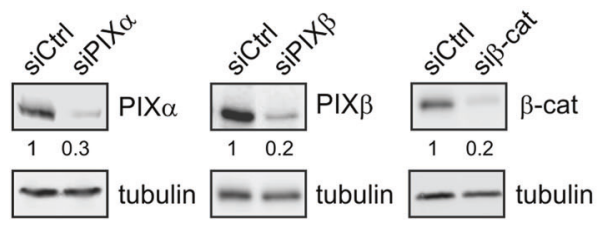

f

f sictrl siPAK2 $\operatorname{siPIX} \alpha \quad \operatorname{siPIX} \beta \quad$ si $\beta$-cat
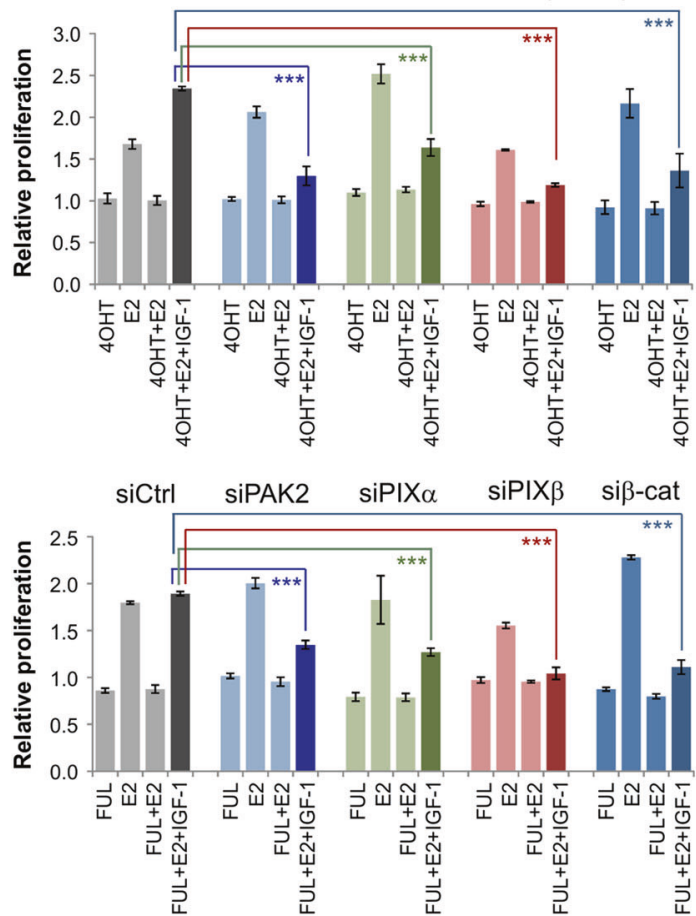

b

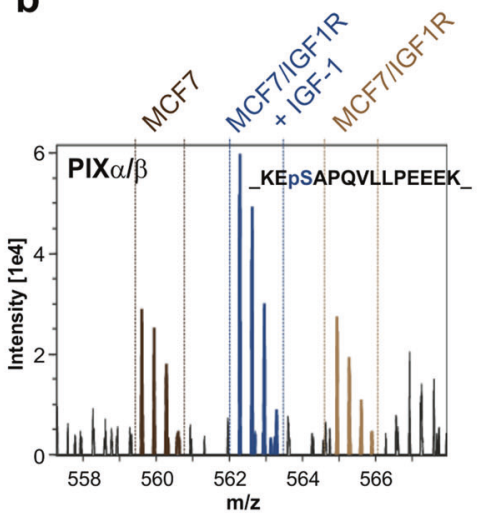

d

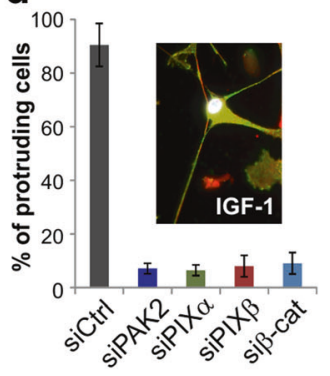

C
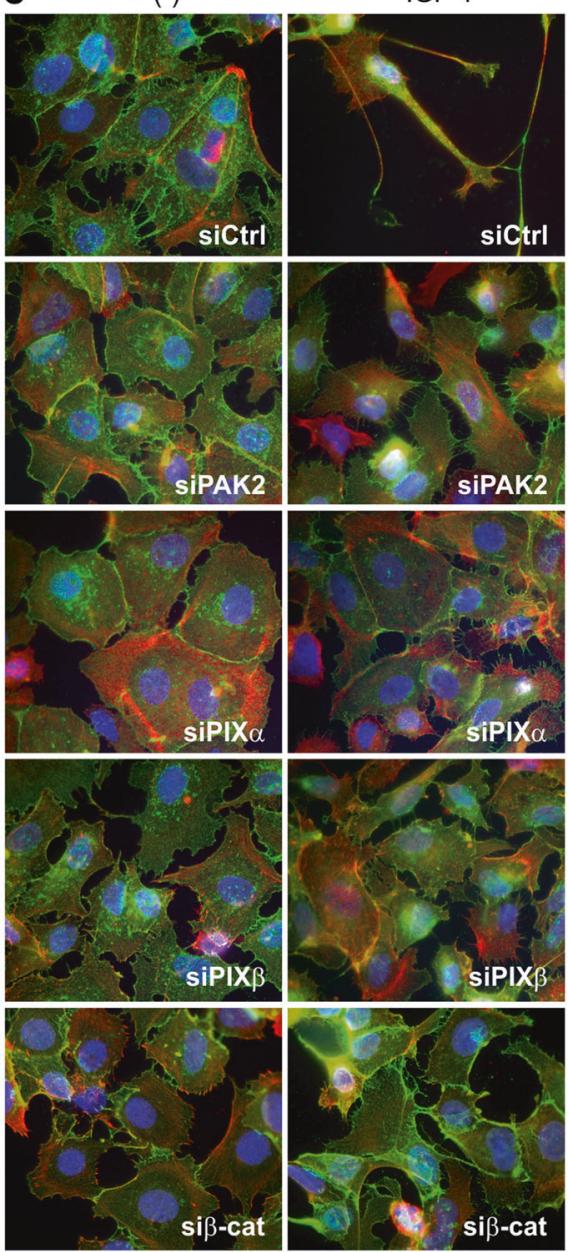

E-cadherin / Actin / Hoechst

g

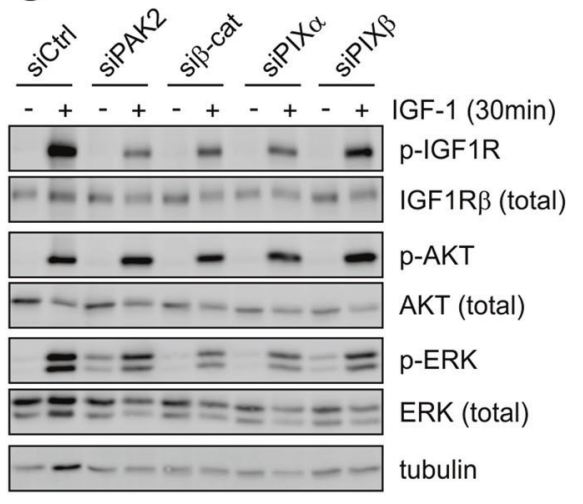

h

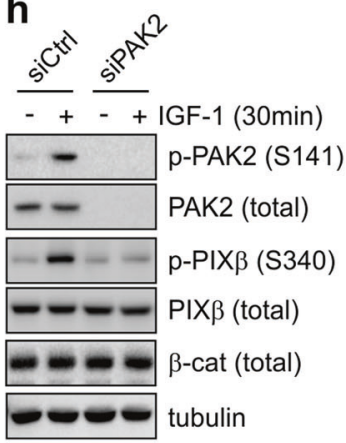

Fig. 7 Involvement of PAK2 survival signaling components PIX $\alpha / \beta$ in IGF1R-mediated antiestrogen resistance. a shPAK2 eliminated IGF-1stimulated scattering phenotype of MCF7/IGF1R cells. IGF-1, $100 \mathrm{ng} /$ $\mathrm{ml}, 30 \mathrm{~min}$. b SILAC spectra graph showed a PIX $\alpha / \beta$ phosphopeptide with high intensity in IGF-1 stimulated MCF7/IGF1R cells. c siPAK2, siPIX $\alpha$, siPIX $\beta$ and $\beta$-catenin ( $\beta$-cat) inhibited cell protrusion of IGF1-stimulated MCF7/IG1R cells, compared to siCtrl. d Quantification of cell protrusion under indicated silencing in response to IGF-1 stimulation. Around 400 cells were counted. Image $\times 60$. e siRNA knockdown of PIX $\alpha, \operatorname{PIX} \beta$ and $\beta$-catenin in MCF7/IGF1R cells. f Targeting of PIX $\alpha$, PIX $\beta$ and $\beta$-catenin re-sensitized MCF7/IGF1R cells to anti-estrogenic effect of 4OHT and FUL, as did siPAK2. Proliferation values were relative to siCtrl under DMSO control. Values were derived from triplicate samples. Data were expressed as mean \pm SD. Significance was determined using a two-sided Student's $t$-test. $* * * P<0.01$. g Similar to siPAK2, siPIX $\alpha, \operatorname{siPIX} \beta$ and si $\beta$-cat did not interfere with phosphorylation of AKT and ERK by IGF-1 stimulation. h PIX $\beta$ phosphorylation downstream PAK2 in IGF-1 stimulation 
A

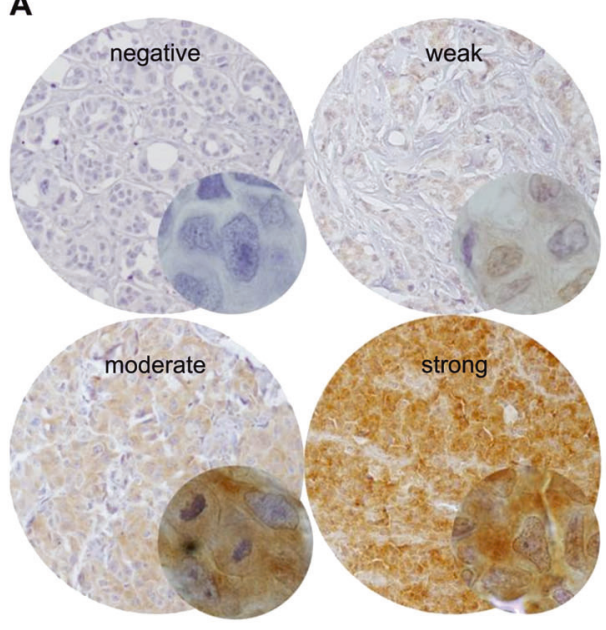

C

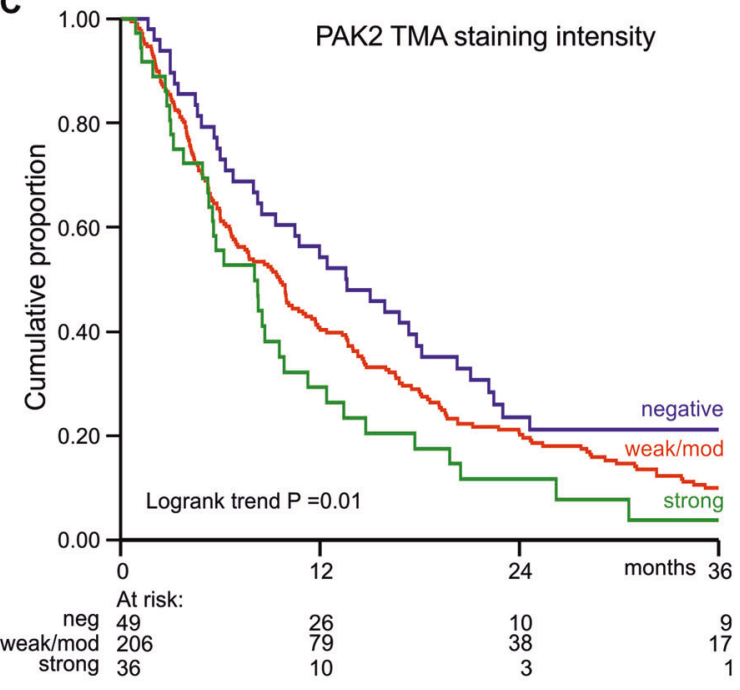

B

\begin{tabular}{|c|c|c|c|c|c|c|c|}
\hline \multirow{2}{*}{$\begin{array}{l}\text { PAK2 protein } \\
\text { intensity }\end{array}$} & \multirow[b]{2}{*}{$N$} & \multicolumn{3}{|c|}{ Univariate analysis } & \multicolumn{3}{|c|}{ Multivariate analysis } \\
\hline & & $\begin{array}{l}\text { Hazard } \\
\text { Ratio }\end{array}$ & $95 \% \mathrm{Cl}$ & $P$ & $\begin{array}{l}\text { Hazard } \\
\text { Ratio }\end{array}$ & $95 \% \mathrm{Cl}$ & $P$ \\
\hline strong versus others & 291 & 1.48 & $1.03-2.13$ & 0.034 & 1.48 & $1.02-2.15$ & 0.039 \\
\hline negative & 49 & 1.00 & & & 1.00 & & \\
\hline weak & 114 & 1.21 & $0.85-1.71$ & 0.289 & 1.21 & $0.84-1.73$ & 0.303 \\
\hline moderate & 92 & 1.30 & $0.91-1.87$ & 0.148 & 1.51 & $1.04-2.21$ & 0.031 \\
\hline strong & 36 & 1.77 & $1.13-2.78$ & 0.013 & 1.87 & $1.81-2.96$ & 0.008 \\
\hline
\end{tabular}

Fig. 8 Relevance of PAK2 expression in primary tumor specimens to poor outcome of metastatic breast cancer patients after first-line tamoxifen. a Representative tissue cores with IHC staining of PAK2 protein, showing negative, weak, moderate and strong cytoplasmic PAK2 staining intensity. Scorings were performed at lower magnification images. b Cox uni and multivariate analysis for PFS of PAK2 protein expression levels in $291 \mathrm{ER}^{+}$primary tumor specimens of

histology, dominant site of relapse, disease-free interval, and the fraction of ER, PgR, and HER2/neu-positive cells (Supplementary Table 2 and Table 3). There was only a significant relationship with the fraction of HER2/neupositive cells. Strong PAK2 staining was significantly associated with PFS in univariate analysis (HR 1.77, 95\% CI 1.13-2.78, $P=0.013$ ) (Figs. 8b, c and Supplementary Fig. $11 \mathrm{~A}$ and $\mathrm{B})$. In multivariate analysis, PAK2 strong staining was related with PFS (HR 1.87, 95\% CI 1.18-2.96, $P=0.008$ ) (Fig. 8b and Supplementary Fig. 11A and B), when compared to tumors with no PAK2 expression and corrected for the traditional predictive factors. PAK2 staining was not related with overall survival (weak: $\mathrm{HR}=1.03,95 \%$ CI $0.71-1.49, P=0.890$; weak/moderate: $\mathrm{HR}=0.99,95 \%$ CI $0.67-1.46, P=0.972$; strong: $\mathrm{HR}=$ $1.19,95 \%$ CI $0.73-1.93, P=0.479)$. These data indicated that high PAK2 expression in MBC patients associates with unfavorable outcome after first-line tamoxifen, strongly suggesting the important clinical correlation of PAK2 to the development of tamoxifen resistance.
MBC patients treated with first-line tamoxifen therapy. c KaplanMeier curve for PFS as function of PAK2 staining intensity in 291 ER + primary tumors of MBC patients treated with first-line tamoxifen. Patients were divided into three groups according to their staining intensity in negative (blue line), in weak/moderate (red line), and in strong staining (green line). Patients at risk at different time points were indicated

\section{Discussion}

Our kinome screen of IGF1R-mediated tamoxifen resistance has defined 10 hits with clinical relevance to $\mathrm{ER}^{+}$ MBC patients treated with first-line tamoxifen monotherapy $[23,24]$. Among tamoxifen resistance suppressors, CDK10 was previously defined as a determinant for tamoxifen resistance [39], while $B M P R 1 B, C D K 5, E I F 2 A K 1$, and $M A P 2 K 5$ are unknown players. The tamoxifen resistance inducer $C H K 1$ has been associated with an impaired tamoxifen response [40], while the implication of $P A K 2$, RPS6KC1, TTK, and TXK in antiestrogen resistance has not been reported yet. Low expression of the suppressors, or enhanced expression of the inducers, as individual markers, might contribute to developing antiestrogen resistance, thereby leading to poor disease outcome. $P A K 2$, as well as RPS6KC1, modulates the proliferation of resistant cells in an IGF1R-signaling dependent manner, suggesting their potency as novel targets in IGF1R-mediated antiestrogen resistance. 
PAK2 belongs to p21 protein (Cdc42/Rac)-activated kinase (PAKs) family that consists of six members. PAK expression and activity are often upregulated in human tumors [28, 41]. PAK1 expression is related to tamoxifen response [42, 43]. We report here that PAK2 expression is significantly predictive for unfavorable outcome in two cohorts of 101 and $291 \mathrm{ER}^{+} \mathrm{MBC}$ patients with first-line of tamoxifen monotherapy, but not in $221 \mathrm{ER}^{+} \mathrm{LNN}$ breast cancer patients without any adjuvant systemic treatment, stressing the clinicopathological correlation of PAK2 to the development of antiestrogen resistance in luminal breast cancer. PAK2 staining intensity was evaluable for $291 \mathrm{ER}^{+}$ primary tumor specimens in our clinical retrospective setting. Recently, a multi-parametric serum marker panel was reported to distinguish breast cancer from healthy control groups. However, the auto-antibodies of this panel, including PAK2, as an individual marker, displayed relative weak discriminatory performance [44]. In our study, almost all tumor cells had some level of PAK2 staining in the majority of tested specimens. Therefore, standard immunoreactive scoring methods combining percentage positive cells with intensity [38] are not appropriate, and also not always applied. For instance, ER and PR in breast cancer are only evaluated for the proportion of positive tumor cells and not for their staining intensities. Rather, we found that analysis of PAK2 staining intensity would be a relevant approach for selection of patients for tamoxifen treatment. Since our cohort differed in the number of cases with regard to staining intensities and the low magnification images showed that strong PAK2 staining was clearly distinguishable from the other intensities, only intensity was further evaluated. Our univariate and multivariate analyses showed that strong PAK2 staining was related to progression free survival, also independent from traditional factors. Thus our study demonstrated strong PAK2 staining in primary breast tumors of especially patients resistant to tamoxifen of the advanced disease setting.

PAKs play pivotal roles in motility, proliferation, mitosis and survival that are required for oncogenesis [28, 45, 46]. Tumor cells with upregulated PAKs tend to become dependent on PAK signaling. It has been uncovered that PAKs interact with downstream $\beta$-catenin to promote growth signaling [37, 47]. PAKs have been placed downstream of PI3K/AKT [45] and upstream of MAPK/ERK canonical cascades [46]. And crosstalk occurs within ERK/ AKT and $\beta$-catenin signaling networks in cell cycle progression [48, 49]. Our results implicate PAK2/PIX and $\beta$ catenin in IGF-1/IGF1R signaling, in a manner that is separate from PI3K/AKT and MAPK/ERK activation. Similar findings have been reported for KRAS-driven proliferation of colon cancer cells [29]. The interaction between PAKs and ERK is complicated. In fibroblast growth factor stimulation, PAK2 is activated downstream of ERK [50], while PAK activity controls ERK activation in the context of platelet derived growth factor but not epidermal growth factor signaling [51]. Moreover, ERK and AKT activity have been reported to be regulated by distinct PAK family members [52]. Clearly, PAKs regulate and mediate signaling cascades in a highly context-dependent manner in response to different stimuli.

Activation of PAK/PIX components conveys oncogenic signaling to promote cell growth and survival, cell migration and invasion, and prevent apoptosis [28, 32-34, 53, 54]. Our results demonstrate that $\mathrm{PAK} 2$ and $\mathrm{PIX} \alpha / \beta$ act downstream of IGF1R signaling to mediate antiestrogen resistance in breast cancer cells. Silencing of PAK2 or $\mathrm{PIX} \alpha / \beta$ attenuates the IGF-1-induced migratory and resistant phenotypes, suggesting that targeting PAK2/PIX survival signaling may provide a novel therapeutic avenue for antiestrogen resistant luminal $\mathrm{ER}^{+}$breast cancers. Future work must be aimed at the identification of pharmacological inhibitors targeting this axis to overcome resistance against antiestrogen therapy in luminal $\mathrm{ER}^{+}$breast cancer.

\section{Materials and methods}

\section{High-throughput kinase and phosphatase siRNA screen and data analysis}

We performed the high-throughput screen on a Biomek FX (Beckman Coulter) liquid handling system under indicated conditions (Supplementary Fig. 2). The primary screen was carried out by use of SMARTpool siRNAs targeting human genome-wide 779 kinases and 193 phosphatases (Dharmacon, Thermo Fisher). Each primary screen plate included positive and negative siRNA controls. In the validation screen, SMARTpool siRNA and single siRNA_1, _2, _3 and $\_4$ that comprise the SMARTpool mix were used to target each candidate hit. For siRNA transfection, 15,000 cells per well were seeded in 96-well plate overnight, transfected for 2 days in replica cell plates (Replica 1 \& 2) with $50 \mathrm{nM}$ siRNA divided from the siRNA transfection plate by use of DharmaFECT 4 transfection reagent (Dharmacon, Thermo Fisher) in 5\% charcoal-dextran treated FBS (CDFBS) starving medium, and proliferated for 4 days under indicated condition. The sulforhodamine B (SRB) colorimetric assay was used as read-out for cell proliferation [14]. Primary screen data were analyzed using an unbiased sample-based analysis [55]. Briefly, raw SRB absorbance values of individual siRNA samples in each plate were transformed into $Z$ scores by the formula " $Z$ score $=$ (individual siRNA sample-mean of all siRNA samples)/standard deviation of all siRNA samples". Validation screen data were assessed with a non-targeting control-based $Z$ score analysis by the formula " $Z$ score $=$ 
(individual siRNA sample-mean of negative controls)/ standard deviation of negative controls". We took $Z>1$ mean $\pm 1.5 \times \mathrm{SDI}$ and $P<0.05$ as significant effect.

\section{Antibodies and compounds, 3D culture, Western blot assay, and immunofluorescence staining}

Mouse antibody specific for PAK2 (\#4825) and rabbit antibody against phospho-PAK1 (Ser144)/PAK2(Ser141) (\#2606) were purchased from Cell Signaling, mouse antibody against E-cadherin (6101810) from BD Transduction, mouse antibody against $\beta$-PIX (611648) from BD Biosciences, and rabbit antibody against $\alpha$-PIX (HPA003578) from Sigma. For other antibodies and compounds, and assays used, they were referred to those as previously described [14].

\section{shRNA knockdown of PAK2}

To establish shRNA-mediated PAK2 knockdown, we used two pLKO.1-puro lentiviral plasmids containing validated human PAK2 shRNA Seq1 (TRCN0000002115; Region: 3UTR; sequence: CCGGCTCTAGGAACCAAAGTGA TTTCTCGAGAAATCACTTTGGTTCCTAGAGTTTTT) or Seq2 (TRCN0000194671; region: CDS; sequence: CCGGCGGGATTTCTTAAATCGATGTCTCGAGACA TCGATTTAAGAAATCCC GTTTTTTG) (Sigma-Aldrich; in collaboration with Dr. Rob Hoeben). The PAK2 shRNA lentiviral plasmid was co-transfected with the packaging constructs pMDLg/pRRE, pRSV-Rev and pCMV-VSV-G by Lipofectamine 2000 (Invitrogen) into human HEK293T cells cultured in 10\% FBS DMEM (GIBCO). The lentiviral particles were freshly harvested and transduced into MCF7/IGF1R cells [14]. The stable shRNAmediated PAK2 knockdown MCF7/IGF1R cell lines were selected with puromycin and named MCF7/IGF1R shPAK2_1 and shPAK2_2, respectively. The shRNA control (shCtrl) pLKO.1-puro plasmid (SHC002, Sigma) targeting no known mammalian DNA was used to establish control cell line MCF7/IGF1R shCtrl.

\section{pCDNA-PAK2 AMAXA transfection}

The pcDNA3.1 $(+)$ vector containing hygromycin resistance gene (pcDNA-hygro) and pcDNA-hygro-PAK2 containing human PAK2 cDNA (pcDNA-PAK2) were kindly provided by Dr. Claude Backendorf (Leiden University, The Netherlands). Transient transfection of the pcDNA-hygro or pcDNA-PAK2 into MCF7/IGF1R shPAK2 cell lines was carried out by use of AMAXA Nucleofector ${ }^{\circledR}$ Kit $\mathrm{V}$ (LonzaBio) under program E-014.

\section{Quantitative SILAC-based phospho-proteomics of MCF7/IGF1R cells under IGF-1 stimulation}

To elucidate the signaling networks assembled by IGF-1R directly after receptor activation in MCF7/IGF1R cells in more detail, we initiated a global phospho-proteomic analysis using stable-isotope labeling by amino acids in cell culture (SILAC) [56, 57]. Briefly, SILAC labeling was performed in parallel, as schemed (Fig. 5a). "Light" labeling with L-[12C6,14N2]lysine (Lys0) and L-[12C6,14N4]arginine (Arg0), "medium" labeling with L-[2H4]lysine (Lys4) and L-[13C6]arginine (Arg6), and "heavy" labeling with L[13C6,15N2]-lysine (Lys8) and L-[13C6,15N4]arginine (Arg10) were used as triple encoding SILAC conditions. After 5 or 7-cell doublings in SILAC culture, the "medium" (Lys4, Arg6) labeled MCF/IGF1R cells were starved in $0.5 \%$ FBS medium overnight and then SFM for 2 hours, followed by 30-min IGF-1 (100 ng/ml) stimulation. "Heavy" (Lys8, Arg10) labeled MCF7/IGF1R cells were left nonstimulated. Parental MCF7 cells were cultured as control with "light" labeling. Then all differentially labeled cells were washed twice with ice-cold $0.5 \times$ PBS (diluted with sterile $\mathrm{H}_{2} \mathrm{O}$ ) and then lysed with a buffer containing $4 \%$ SDS, $100 \mathrm{mM}$ Tris- $\mathrm{HCl}(\mathrm{pH} 7.8)$ and $0.1 \mathrm{M}$ DTT in $\mathrm{H} 2 \mathrm{O}$ and harvested into sterile polypropylene reaction tubes. For phosphopeptide identification and quantitation by HighPerformance Liquid Chromatography (HPLC) and Mass Spectrometry (MS), equal amount of protein (1:1:1) from each SILAC condition was mixed following trypsin digestion according to the filter aided sample preparation protocol [58] and peptide fractionation by strong cation exchange chromatography, followed by titanium-dioxide phosphorpeptide enrichment [59]. MS analysis was performed on the LTQ-Orbitrap Velos mass spectrometer with high-energy collisional dissociation fragmentation. Raw MS files were analyzed with MaxQuant.

\section{Flow cytometry cell cycle assay}

Cells $(200,000 /$ well $)$ were seeded in 12 -w cell plate overnight, starved for 2 days in 5\% CDFBS medium, and then treated under drug conditions as indicated. The time after 2day starvation and before drug treatment was considered to be Day 0 . Cells were then harvested after 4-day treatment, fixed with cold $100 \%$ ethanol and labeled in staining solution containing $10 \mu \mathrm{M}$ propidium iodide (PI) and $50 \mathrm{ng} /$ ml RNase A (BD Cycletest ${ }^{\mathrm{TM}}$ Plus DNA Reagent Kit, BD Biosciences). Cells were analyzed by use of FACSCanto II flow cytometer (BD Biosciences). The percentage of cells in G0/G1, S and G2/M phases were determined from 10,000 ungated cells (cell events) using the FACSDiva Software v6.1.2 (BD Biosciences). 


\section{Annexin V apoptosis assay}

To detect apoptosis, a live cell imaging of Annexin VAlexa633 labeling was performed in real time, as described previously [60]. Briefly, 10,000 cells/well were seeded in 96-well plate. Following 2-day starvation in 5\% CDFBS medium, cells were treated as indicated and labeled with Annexin V-Alexa633 $(250 \mathrm{ng} / \mathrm{ml})$ that conjugates to phosphatidyl serine on the membranes of apoptotic cells. At the time points of day $0,1,2,3$, and 4, the Annexin V in-taken cells were captured under Cy5 channel and the whole cell population was imaged under transmitted light by use of BD pathway 855 imager (Becton Dickinson). Simultaneously, the nuclei of live cells were stained with DNA dye Hoechst $33342(200 \mathrm{ng} / \mathrm{ml})$ and imaged for cell density. The number of Annexin V positive cells and the total cell number in each well were quantified using Image Pro (Media Cybernetics, Bethesda, MD, USA). Annexin V apoptosis fraction was calculated by normalization of Annexin $\mathrm{V}$ positive cells to the total cell number.

\section{Experimental statistical analysis}

Each average value was derived from triplicate experimental samples. Statistical analysis of all experimental data was performed using a two-sided Student's $t$-test. Data were expressed as mean \pm SD. Significance was set at $P<0.05$.

\section{Ethics statement}

The retrospective studies were approved by the medical ethics committee of the Erasmus MC Rotterdam, the Netherlands (MEC 02.953) and included coded fresh frozen and/or formalin-fixed, paraffin-embedded primary breast tumor tissue specimens from patients with primary operable breast cancer between 1985 and 2000. It was carried out according the Code of Conduct of the Federation of Medical Scientific Societies in The Netherlands (http://www.fmwv. nl) and reported, wherever possible, following the REMARK guidelines [61].

\section{Patients gene expression data}

Patients with primary operable breast cancer were included in study who subsequently developed MBC treated with first-line tamoxifen and for which detailed clinical followup was available. For the gene expression microarray analyses, a cohort of 101 patients was investigated. This cohort has already been described by us previously [24]. The tissue microarray analyses used a cohort of 250 patients that has been described by us [38], but includes now 41 additional patients. Briefly, for 291 of the 378 available patients ERpositive tumor core biopsies of the breast TMA were selected for further analyses. Of these 291 patients, 128 (44\%) patients underwent breast conserving surgery and 163 modified mastectomy (56\%). Median follow-up time after start therapy for patients alive was 42 months (range 6-188 months). All patients received first-line tamoxifen, and of these were 174 patients $(60 \%)$ hormone-naïve and 117 patients (40\%) received adjuvant chemotherapy. Response to therapy was observed in 181 patients $(62 \%)$ whereas 110 patients $(38 \%)$ did not have clinical benefit. The patients with response included 7 patients (4\%) with complete response, 50 patients $(28 \%)$ with partial response and 124 patients $(68 \%)$ with stable disease for more than six months.

\section{Patient tissue microarrays and immunohistological evaluation}

Tissue microarrays of all formalin-fixed, paraffin-embedded primary breast tumor specimens were prepared and immunohistochemically stained according to the procedures described previously [38]. The staining was performed with the primary mouse monoclonal antibody against PAK2 and incubated for 1 hour (1:50 dilution) after $40 \mathrm{~min}$ antigen retrieval at pH9.0. Subsequently, TMA slides were incubated with a secondary antibody and staining was visualized using diaminobenzidine. PAK2 protein staining was scored for quantity and intensity by two independent observers, using Slidepath software (Leica Biosystems, Dublin). For the web-based scoring, stained TMAs were digitalized by a Hamamatsu Nanozoomer 2.0 HT (Hamamatsu photonics, Hamamatsu, Japan at magnification $\times 20(0.45 \mu \mathrm{m} /$ pixel resolution). Staining was grouped according to standardized categories for the estimated percentage of PAK2 positive cells $(0 \%, 1-4 \%$ positive cells, $5-10 \%, 11-20 \%, 21-30 \%$, $31-40 \%, 41-50 \%, 51-99 \%, 100 \%)$ and staining intensity as exemplified in Fig. 8a (negative, weak, moderate, strong). More information for patients, ethics statement, data analysis and statistical methods, see supplemental information.

\section{Patient data analysis and statistics}

Statistical analyses were performed with STATA statistical package, release 13.0 (STATA Corp., College Station, TX, USA). Pearson's chi-squared, Mann-Whitney $U$ tests, and Fisher's exact test were used to investigate the association between PAK2 protein expression and clinicopathological factors. HR with $95 \%$ CI was computed by the Cox proportional hazard model to assess the relationship between PAK2 protein expression and PFS after first-line therapy with tamoxifen. The endpoint PFS was defined as the time elapsed between start of tamoxifen therapy and the first detection of disease progression or death, whatever came first during treatment [38]. A multivariate analysis was 
performed to determine whether PAK2 expression had predictive value and was independent when added to base model of clinicopathological factors. Survival curves were generated by the Kaplan-Meier method. The log-rank test and log-rank test for trends were used to test for differences between survival curves. The $P$-values were two sided and $P<0.05$ was considered as statistically significant.

Acknowledgements This study was supported by the research project of Top Institute Pharma (Leiden, The Netherlands) "Nuclear Receptors in Targeted Cancer Therapy" (T3-107) and the research project of Dutch Cancer Society "Novel Protein Kinase Targets in Receptor Tyrosine Kinase-mediated Drug Resistant Breast Cancer" (UL-20115124).

Author contributions YZ, JHNM, MPHMJ, and BvdW designed research. RS, YZ, and EHJD designed automated high-throughput screening. YZ performed screening and analyzed screening data. TG, $\mathrm{CP}, \mathrm{YZ}$, and MdG contributed to SILAC phospho-proteomics and data analysis. JAH, MMT, MPL, CHMvD, JWMM, EMJJB, and MPHMJ contributed to clinical evaluations and analyses. YZ, LW, JH, and MM carried out experimental assays. YZ, MPHMJ, and BvdW wrote the manuscript.

\section{Compliance with ethical standards}

Conflict of interest The authors declare that they have no competing interests.

\section{References}

1. Osborne CK, Yochmowitz MG, Knight WA 3rd, McGuire WL. The value of estrogen and progesterone receptors in the treatment of breast cancer. Cancer 1980;46:2884-8.

2. Arimidex, Tamoxifen, Alone or in Combination (ATAC) Trialists' Group, Forbes JF, Cuzick J, Buzdar A, Howell A, Tobias JS. et al. Effect of anastrozole and tamoxifen as adjuvant treatment for early-stage breast cancer: 100-month analysis of the ATAC trial. Lancet Oncol 2008;9:45-53.

3. Jaiyesimi IA, Buzdar AU, Decker DA, Hortobagyi GN. Use of tamoxifen for breast cancer: twenty-eight years later. J Clin Oncol: J Am Soc Clin Oncol 1995;13:513-29.

4. Cleator SJ, Ahamed E, Coombes RC, Palmieri C. A 2009 update on the treatment of patients with hormone receptor-positive breast cancer. Clin Breast Cancer 2009;9(Suppl 1):S6-S17.

5. Musgrove EA, Sutherland RL. Biological determinants of endocrine resistance in breast cancer. Nat Rev Cancer 2009;9:631-43.

6. Ring A, Dowsett M. Mechanisms of tamoxifen resistance. Endocr Relat Cancer 2004;11:643-58.

7. Shou J, Massarweh S, Osborne CK, Wakeling AE, Ali S, Weiss $\mathrm{H}$, et al. Mechanisms of tamoxifen resistance: increased estrogen receptor-HER2/neu cross-talk in ER/HER2-positive breast cancer. J Natl Cancer Inst 2004;96:926-35.

8. Jin K, Kong X, Shah T, Penet MF, Wildes F, Sgroi DC, et al. The HOXB7 protein renders breast cancer cells resistant to tamoxifen through activation of the EGFR pathway. Proc Natl Acad Sci USA 2012;109:2736-41.

9. Moerkens M, Zhang Y, Wester L, van de Water B, Meerman JH. Epidermal growth factor receptor signalling in human breast cancer cells operates parallel to estrogen receptor alpha signalling and results in tamoxifen insensitive proliferation. BMC Cancer 2014; 14:283.
10. Jin K, Park S, Teo WW, Korangath P, Cho SS, Yoshida T, et al. HOXB7 Is an ERalpha cofactor in the activation of HER2 and multiple ER target genes leading to endocrine resistance. Cancer Discov 2015;5:944-59.

11. Meng S, Tripathy D, Shete S, Ashfaq R, Haley B, Perkins S, et al. HER-2 gene amplification can be acquired as breast cancer progresses. Proc Natl Acad Sci USA 2004;101:9393-8.

12. Fox EM, Miller TW, Balko JM, Kuba MG, Sanchez V, Smith RA, et al. A kinome-wide screen identifies the insulin/IGF-I receptor pathway as a mechanism of escape from hormone dependence in breast cancer. Cancer Res 2011;71:6773-84.

13. Winder T, Giamas G, Wilson PM, Zhang W, Yang D, Bohanes P, et al. Insulin-like growth factor receptor polymorphism defines clinical outcome in estrogen receptor-positive breast cancer patients treated with tamoxifen. Pharm J 2014;14:28-34.

14. Zhang Y, Moerkens M, Ramaiahgari S, de Bont H, Price L, Meerman J, et al. Elevated insulin-like growth factor 1 receptor signaling induces antiestrogen resistance through the MAPK/ERK and PI3K/Akt signaling routes. Breast Cancer Res 2011;13:R52.

15. Arnaldez FI, Helman LJ Targeting the insulin growth factor receptor 1. Hematol Oncolo Clin North Am 2012; 26: 527-42, viiviii.

16. Law JH, Habibi G, Hu K, Masoudi H, Wang MY, Stratford AL, et al. Phosphorylated insulin-like growth factor-i/insulin receptor is present in all breast cancer subtypes and is related to poor survival. Cancer Res 2008;68:10238-46.

17. Luey BC, May FE. Insulin-like growth factors are essential to prevent anoikis in oestrogen-responsive breast cancer cells: importance of the type I IGF receptor and PI3-kinase/Akt pathway. Mol Cancer 2016;15:8.

18. Sachdev D. Regulation of breast cancer metastasis by IGF signaling. J Mammary Gland Biol Neoplasia 2008;13:431-41.

19. Werner H, Bruchim I. The insulin-like growth factor-I receptor as an oncogene. Arch Physiol Biochem 2009;115:58-71.

20. Heskamp S, Boerman OC, Molkenboer-Kuenen JD, Wauters CA, Strobbe LJ, Mandigers CM, et al. Upregulation of IGF-1R expression during neoadjuvant therapy predicts poor outcome in breast cancer patients. Plos One 2015;10:e0117745.

21. Gonzalez-Malerva L, Park J, Zou L, Hu Y, Moradpour Z, Pearlberg $\mathrm{J}$, et al. High-throughput ectopic expression screen for tamoxifen resistance identifies an atypical kinase that blocks autophagy. Proc Natl Acad Sci USA 2011;108:2058-63.

22. Mendes-Pereira AM, Sims D, Dexter T, Fenwick K, Assiotis I, Kozarewa I, et al. Genome-wide functional screen identifies a compendium of genes affecting sensitivity to tamoxifen. Proc Natl Acad Sci USA 2012;109:2730-5.

23. Jansen MP, Foekens JA, van Staveren IL, Dirkzwager-Kiel MM, Ritstier K, Look MP, et al. Molecular classification of tamoxifenresistant breast carcinomas by gene expression profiling. J Clin Oncol 2005;23:732-40.

24. Kok M, Linn SC, Van Laar RK, Jansen MP, van den Berg TM, Delahaye LJ, et al. Comparison of gene expression profiles predicting progression in breast cancer patients treated with tamoxifen. Breast Cancer Res Treat 2009;113:275-83.

25. Wang Y, Klijn JG, Zhang Y, Sieuwerts AM, Look MP, Yang F, et al. Gene-expression profiles to predict distant metastasis of lymph-node-negative primary breast cancer. Lancet 2005;365:671-9.

26. Nheu T, He H, Hirokawa Y, Walker F, Wood J, Maruta H. PAK is essential for RAS-induced upregulation of cyclin D1 during the G1 to S transition. Cell Cycle 2004;3:71-74.

27. Puto LA, Pestonjamasp K, King CC, Bokoch GM. p21-activated kinase 1 (PAK1) interacts with the Grb2 adapter protein to couple to growth factor signaling. J Biol Chem 2003;278:9388-93. 
28. Radu M, Semenova G, Kosoff R, Chernoff J. PAK signalling during the development and progression of cancer. Nat Rev Cancer 2014;14:13-25.

29. Tabusa H, Brooks T, Massey AJ. Knockdown of PAK4 or PAK1 inhibits the proliferation of mutant KRAS colon cancer cells independently of RAF/MEK/ERK and PI3K/AKT signaling. Mol Cancer Res: MCR 2013;11:109-21.

30. Wang Z, Fu M, Wang L, Liu J, Li Y, Brakebusch C, et al. p21activated kinase 1 (PAK1) can promote ERK activation in a kinase-independent manner. J Biol Chem 2013;288:20093-9.

31. Ong CC, Jubb AM, Haverty PM, Zhou W, Tran V, Truong T, et al. Targeting p21-activated kinase 1 (PAK1) to induce apoptosis of tumor cells. Proc Natl Acad Sci USA 2011;108:7177-82.

32. Frank SR, Bell JH, Frodin M, Hansen SH. A betaPIX-PAK2 complex confers protection against Scrib-dependent and cadherinmediated apoptosis. Curr Biol 2012;22:1747-54.

33. Menges CW, Sementino E, Talarchek J, Xu J, Chernoff J, Peterson JR, et al. Group I p21-activated kinases (PAKs) promote tumor cell proliferation and survival through the AKT1 and RafMAPK pathways. Mol Cancer Res 2012;10:1178-88.

34. Santiago-Medina M, Gregus KA, Gomez TM. PAK-PIX interactions regulate adhesion dynamics and membrane protrusion to control neurite outgrowth. J Cell Sci 2013;126:1122-33.

35. Zhou L, Ercolano E, Ammoun S, Schmid MC, Barczyk MA, Hanemann CO. Merlin-Deficient Human Tumors Show Loss of Contact Inhibition and Activation of Wnt/beta-Catenin Signaling Linked to the PDGFR/Src and Rac/PAK Pathways. Neoplasia 2011;13:1101-U1117.

36. Ye P, Hu Q, Liu H, Yan Y, D'Ercole AJ. beta-catenin mediates insulin-like growth factor-I actions to promote cyclin D1 mRNA expression, cell proliferation and survival in oligodendroglial cultures. Glia 2010;58:1031-41.

37. Arias-Romero LE, Villamar-Cruz O, Huang M, Hoeflich KP, Chernoff J. Pak1 Kinase Links ErbB2 to beta-Catenin in Transformation of Breast Epithelial Cells. Cancer Res 2013;73:3671-82.

38. Reijm EA, Timmermans AM, Look MP, Meijer-van Gelder ME, Stobbe CK, van Deurzen $\mathrm{CH}$, et al. High protein expression of EZH2 is related to unfavorable outcome to tamoxifen in metastatic breast cancer. Ann Oncol 2014;25:2185-90.

39. Iorns E, Turner NC, Elliott R, Syed N, Garrone O, Gasco M, et al. Identification of CDK10 as an important determinant of resistance to endocrine therapy for breast cancer. Cancer Cell 2008;13:91-104.

40. Lundgren K, Holm K, Nordenskjold B, Borg A, Landberg G. Gene products of chromosome $11 \mathrm{q}$ and their association with CCND1 gene amplification and tamoxifen resistance in premenopausal breast cancer. Breast Cancer Res 2008;10:R81.

41. Gao C, Ma T, Pang L, Xie R. Activation of P21-activated protein kinase 2 is an independent prognostic predictor for patients with gastric cancer. Diagn Pathol 2014;9:55.

42. Holm C, Rayala S, Jirstrom K, Stal O, Kumar R, Landberg G. Association between Pak1 expression and subcellular localization and tamoxifen resistance in breast cancer patients. J Natl Cancer Inst 2006;98:671-80.

43. Kok M, Zwart W, Holm C, Fles R, Hauptmann M, Van't Veer LJ, et al. PKA-induced phosphorylation of ERalpha at serine 305 and high PAK1 levels is associated with sensitivity to tamoxifen in ER-positive breast cancer. Breast Cancer Res Treat 2011;125:1-12.

44. Lacombe J, Mange A, Bougnoux AC, Prassas I, Solassol J. A multiparametric serum marker panel as a complementary test to mammography for the diagnosis of node-negative early-stage breast cancer and DCIS in young women. Cancer Epidemiol Biomark Prev 2014;23:1834-42.

45. Kumar R, Gururaj AE, Barnes CJ. p21-activated kinases in cancer. Nat Rev Cancer 2006;6:459-71.

46. Molli PR, Li DQ, Murray BW, Rayala SK, Kumar R. PAK signaling in oncogenesis. Oncogene 2009;28:2545-55.

47. Radu M, Semenova G, Kosoff R, Chernoff J. PAK signalling during the development and progression of cancer. Nat Rev Cancer 2014;14:13-25.

48. Padala RR, Karnawat R, Viswanathan SB, Thakkar AV, Das AB, Cancerous perturbations within the ERK, PI3K/Akt, and Wnt/ beta-catenin signaling network constitutively activate interpathway positive feedback loops. Mol Biosyst. 2017;13:830-40.

49. Vadlakonda L, Pasupuleti M, Pallu R. Role of PI3K-AKT-mTOR and Wnt signaling pathways in transition of G1-S phase of cell cycle in cancer cells. Front Oncol 2013;3:85.

50. Shin EY, Shin KS, Lee CS, Woo KN, Quan SH, Soung NK, et al. Phosphorylation of $\mathrm{p} 85$ beta PIX, a Rac/Cdc42-specific guanine nucleotide exchange factor, via the Ras/ERK/PAK2 pathway is required for basic fibroblast growth factor-induced neurite outgrowth. J Biol Chem 2002;277:44417-30.

51. Beeser A, Jaffer ZM, Hofmann C, Chernoff J. Role of group A p21-activated kinases in activation of extracellular-regulated kinase by growth factors. J Biol Chem 2005;280:36609-15.

52. Wang Z, Pedersen E, Basse A, Lefever T, Peyrollier K, Kapoor S, et al. Rac1 is crucial for Ras-dependent skin tumor formation by controlling Pak1-Mek-Erk hyperactivation and hyperproliferation in vivo. Oncogene 2010;29:3362-73.

53. Feng Q, Baird D, Yoo S, Antonyak M, Cerione RA. Phosphorylation of the cool-1/beta-Pix protein serves as a regulatory signal for the migration and invasive activity of Src-transformed cells. J Biol Chem 2010;285:18806-16.

54. Hsu RM, Tsai MH, Hsieh YJ, Lyu PC, Yu JS. Identification of MYO18A as a novel interacting partner of the PAK2/betaPIX/ GIT1 complex and its potential function in modulating epithelial cell migration. Mol Biol Cell 2010;21:287-301.

55. Birmingham A, Selfors LM, Forster T, Wrobel D, Kennedy CJ, Shanks E, et al. Statistical methods for analysis of high-throughput RNA interference screens. Nat Method 2009;6:569-75.

56. Mann M. Functional and quantitative proteomics using SILAC. Nat Rev Mol Cell Biol 2006;7:952-8.

57. Ong SE, Blagoev B, Kratchmarova I, Kristensen DB, Steen H, Pandey A, et al. Stable isotope labeling by amino acids in cell culture, SILAC, as a simple and accurate approach to expression proteomics. Mol Cell Proteom 2002;1:376-86.

58. Wisniewski JR, Zougman A, Nagaraj N, Mann M. Universal sample preparation method for proteome analysis. Nat Method 2009;6:359-62.

59. Macek B, Mann M, Olsen JV. Global and site-specific quantitative phosphoproteomics: principles and applications. Annu Rev Pharmacol Toxicol 2009;49:199-221.

60. Puigvert JC, de Bont H, van de Water B, Danen EH Highthroughput live cell imaging of apoptosis. Current protocols in cell biology/editorial board, Juan S Bonifacino [et al.]2010; Chapter 18: Unit 18 10 11-13.

61. McShane LM, Altman DG, Sauerbrei W, Taube SE, Gion M, Clark GM, et al. REporting recommendations for tumor MARKer prognostic studies (REMARK). Breast Cancer Res Treat 2006;100:229-35. 